\title{
Sustainable Alkylation of Nitriles with Alcohols by Manganese Catal- ysis
}

\author{
Jannik C. Borghs, ${ }^{\dagger}$ Mai Anh Tran, ${ }^{\dagger}$ Jan Sklyaruk, ${ }^{\dagger}$ Magnus Rueping, ${ }^{\dagger *}$ and Osama El-Sepelgy ${ }^{\dagger *}$ \\ $\dagger$ Institute of Organic Chemistry, RWTH Aachen University, Landoltweg 1, 52074 Aachen, Germany \\ $\$$ KAUST Catalysis Center (KCC), King Abdullah University of Science and Technology (KAUST), Thuwal 23955-6900, \\ Saudi Arabia
}

\begin{abstract}
A general and chemoselective catalytic alkylation of nitriles using a homogenous non-precious manganese catalyst is presented. This alkylation reaction uses naturally abundant alcohols and readily available nitriles as coupling partners. The reaction tolerates a wide range of functional groups and heterocyclic moieties, efficiently providing useful cyanoalkylated products with water as the only side product. Importantly, methanol can be used as a $C 1$ source and the chemoselective $C$-methylation of nitriles is achieved. The mechanistic investigations support the multiple role of the metal-ligand manganese catalyst; the dehydrogenative activation of the alcohol; $\alpha-\mathrm{C}-\mathrm{H}$ activation of the nitrile; and hydrogenation of the in-situ formed unsaturated intermediate.
\end{abstract}

\section{INTRODUCTION}

The construction of $\mathrm{C}-\mathrm{C}$ bonds via the hydrogen autotransfer strategy is of particular academic and industrial interest. The key motivation for this strategy is the application of alcohol feedstock as a benign alkylating reagent, ${ }^{1}$ while liberating water as the sole by-product. Thus, the procedure eliminates the need for mutagenic alkyl halides and avoids the production of copious waste. To date the majority of appropriate catalytic systems are based on noble metals including $\mathrm{Ru}, \mathrm{Rh}, \mathrm{Ir}, \mathrm{Pd}$ and $\mathrm{Pt}^{2}{ }^{2}$ The low availability of these precious metals has triggered the development of alternative catalytic systems based on earth abundant metals. ${ }^{3}$ In this context, progress has been made in the alkylation of ketones, ${ }^{4}$ alcohols, ${ }^{5}$ esters, ${ }^{6}$ and amides ${ }^{6}$ using base metal catalysts. In contrast, the alkylation of nitriles is more challenging due to the sensitivity of the cyano group towards the activated hydrogen and the water side product. In more detail, the metal catalyst can transfer hydrogen from the alcohol to the nitriles to form primary amines and aldehydes, ${ }^{7}$ and these can subsequently be converted to secondary amines ${ }^{8}$ or amides. ${ }^{9}$ However, water might result in the hydrolysis of the nitrile functionality to the corresponding amide. ${ }^{10}$ In addition, the alkylation of nitriles with alcohols often leads to a mixture of the alkylnitrile product along with the corresponding olefin intermediate, ${ }^{11}$ giving rise to critical selectivity issues. Thus, until recently, the direct alkylation of nitriles with alcohols was only known with precious metal catalysis such as $\mathrm{Ru},{ }^{12} \mathrm{Ir},{ }^{13} \mathrm{Rh},{ }^{14} \mathrm{Os}{ }^{15}$ and $\mathrm{Pd} .{ }^{16}$

Our interest in the development of an improved synthesis of alkylnitriles is due to their great importance as valuable intermediates in the fine chemical industry. Importantly, the cyanoalkyl moiety is also ubiquitous in different natural products and pharmaceuticals. ${ }^{17}$ Very recently, Wang and coworkers have reported an interesting iron catalysed alkylation of nitriles with primary alcohols, by applying stoichiometric amount of strong base $(\mathrm{NaOH}){ }^{18}$ Pursuant to our interest in developing sustainable transformations based on nonprecious metal catalysis, ${ }^{19}$ and inspired by the recent advances in manganese catalysis, ${ }^{20-22}$ we herein report highly reactive and selective manganese catalysed alkylation of non-activated nitriles with a broad range of alcohols (Scheme 1). A related study has also been reported by Maji et. al. ${ }^{23}$ In our study we focused on a catalytic system that utilizes a bench stable catalyst which can easily be prepared in one step from the commercially available and air stable MACHO ligand and $\mathrm{Mn}(\mathrm{CO})_{5} \mathrm{Br}$. Interestingly, the presented catalytic system is not limited to the long-chain alcohols as the more challenging $C$ alkylation can also be achieved with methanol as $\mathrm{C} 1$-source. Furthermore, the manganese catalyst does not require any sensitive reagents for activation and is simply activated with catalytic amounts of suitable base.

Scheme 1. Manganese Catalyzed Alkylation of Nitriles with Alcohols

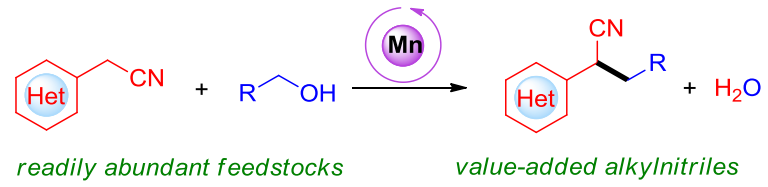

\section{RESULTS AND DISCUSSION}

In order to develop the base metal catalyzed alkylation reaction we started to investigate the reaction between phenylacetonitrile (1a) and the $n$-butanol (2a) applying different manganese catalysts (Table 1). Initially, we screened the catalytic activity of the different manganese complexes Mn-1 to $\mathbf{M n - 3}$ in toluene using $t$-BuOK as the catalyst activator. The pyridyl-based PNP complex Mn-1 ${ }^{22 j}$ led to unsatisfactory results providing only a trace amount of the product (Table 1 , entry 1). However, in the presence of the complex Mn-2, ${ }^{22 \mathrm{k}}$ bearing an aliphatic $\mathrm{NH}$ group, excellent conversion was observed and $86 \%$ of the desired product 3a along with $11 \%$ of the unsaturated olefin 3a'(Table 1, entry 2) was obtained. Use of the Mn-PNN pincer complex $\mathbf{M n - \mathbf { 3 } ^ { 2 0 b }}$ (Table 1, entry 3) provided similar results. Due to the facile access of $\mathbf{M n - 2}$ and the availability of the ligand, we decided to further optimize the model reaction using $\mathbf{M n - 2}$ in combination with different bases and solvents. Running the reaction using equimolar amounts of the manganese and the base, resulted in $66 \%$ con- 
version and 47\% combined yield of 3a and 3a' (Table 1, entry 4). Using polar solvents such as $t$-amyl alcohol, 1,4-Dioxane or 2-Me-THF, did not lead to improved results (Table 1, entries 5-7). In addition, we tested various bases including $\mathrm{KOH}$, $\mathrm{K}_{2} \mathrm{CO}_{3}$ and $\mathrm{Cs}_{2} \mathrm{CO}_{3}$ (Table 1, entries 8-10). From these experiments the best results were obtained using $\mathrm{Cs}_{2} \mathrm{CO}_{3}$ and the desired product was obtained in $79 \%$ yield along with $8 \%$ of the alkenyl nitrile 3a'. Pleasingly, slightly increasing the base loading to $10 \mathrm{~mol} \%$ led to quantitative yield with complete chemoselectivity (Table 1, entry 11).

Table 1. Optimization of the Reaction Conditions. ${ }^{a}$

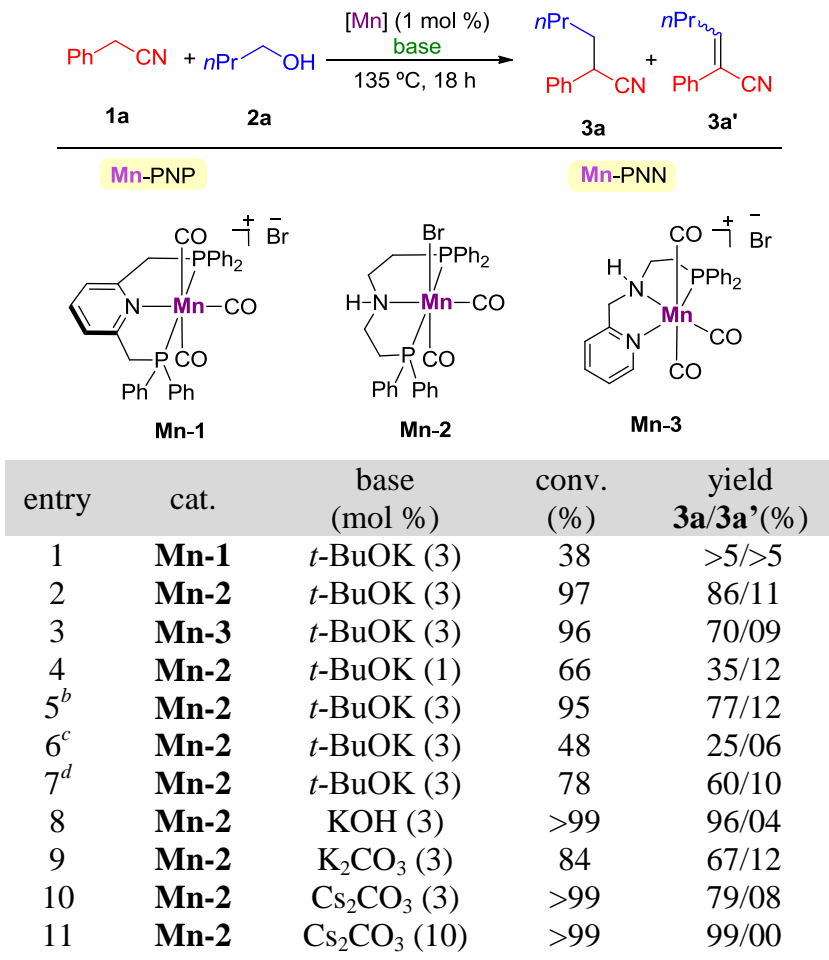

${ }^{a}$ Reaction conditions: 1a $(0.5 \mathrm{mmol})$, 2a $(1 \mathrm{mmol})$, [Mn] $(0.005 \mathrm{mmol})$ and base in toluene $(0.5 \mathrm{~mL})$ at $135{ }^{\circ} \mathrm{C}$ in a glass tube under an inert atmosphere for $18 \mathrm{~h}$. Conversions and yields were determined by GC analysis of the crude reaction mixture using mesytilene as an internal standard. ${ }^{b}$ Reaction in $t$-amyl alcohol. ${ }^{c}$ Reaction in 2-Me THF. ${ }^{d}$ Reaction in 1,4-dioxane.

Having established the optimized reaction conditions, we next investigated the generality of this protocol by exploring the scope of the nitrile alkylation partner (Scheme 2A). To our delight, in all cases the reaction was accomplished within $18 \mathrm{~h}$ using $1 \mathrm{~mol} \%$ of the bench stable catalyst Mn-2. Notably, a diverse range of substituted benzyl cyanides can be used to yield the $C$-alkylated nitriles derivatives $\mathbf{3 b}-\mathbf{3} \mathbf{g}$ in very good yields and with excellent chemoselectivity. It is important to highlight that the benzonitrile moiety was tolerated to give the desired product $\mathbf{3 h}$. Importantly, heterocycle containing benzylic nitriles, such as $\mathbf{3 i}$ and $\mathbf{3 j}$ can also be efficiently alkylated.

We next decided to explore the scope of primary alcohols as alkylating agents (Scheme 2B). Importantly, a large array of benzyl alcohols bearing electron withdrawing and electron donating groups in different positions could be applied to give
Scheme 2. Manganese Catalyzed Alkylation of Nitriles with Different Alcohols.

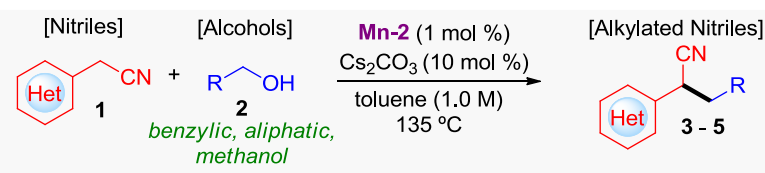

A) Nitrile coupling partners ${ }^{a}$
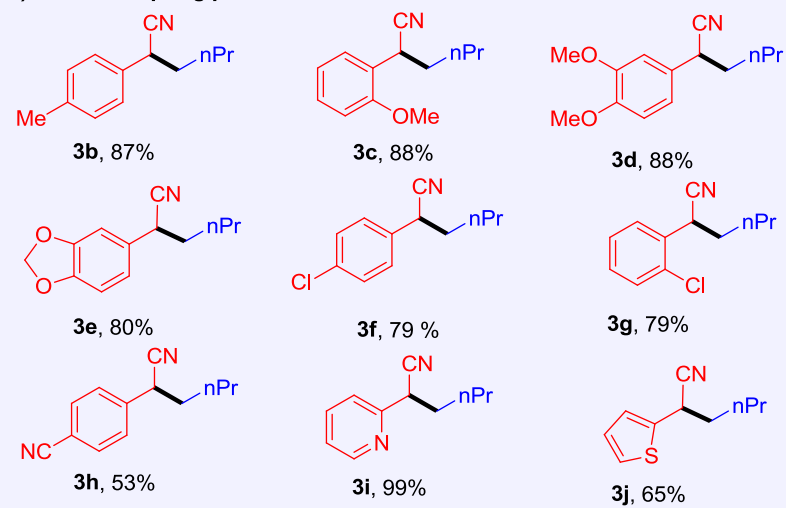

B) Alcohol coupling partners
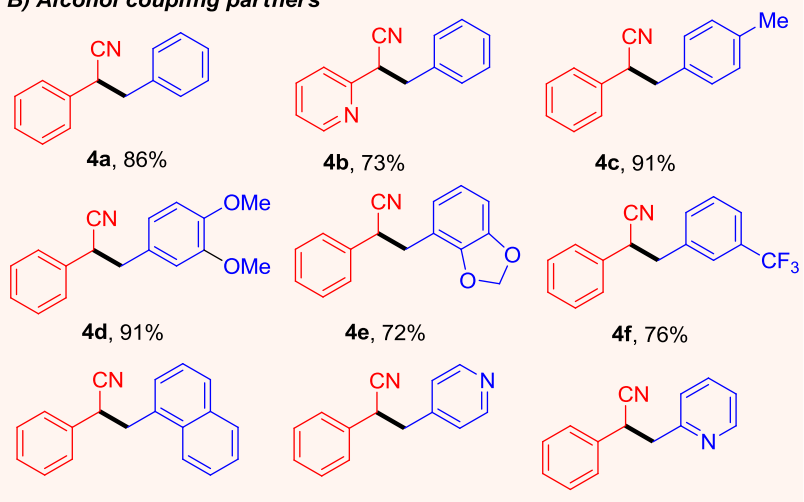

$\begin{array}{lll}\mathbf{4 g}, 83 \% & \mathbf{4 h}, 70 \% & \mathbf{4 i}, 47 \%\end{array}$

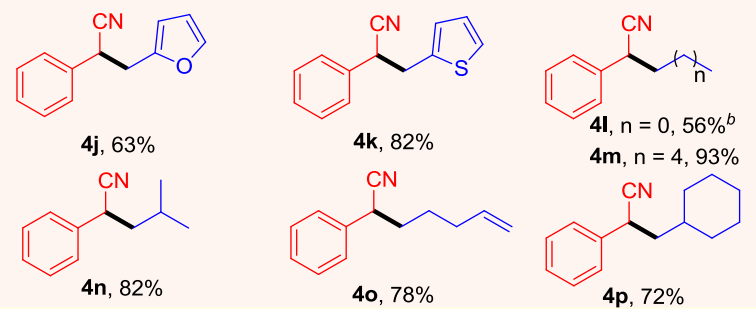

C) Methanol as coupling partner ${ }^{c}$
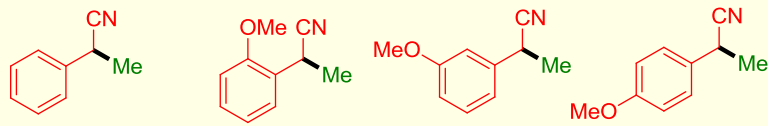

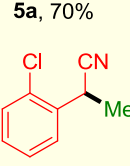

5e, $61 \%$

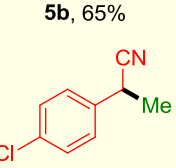

5f, $69 \%$

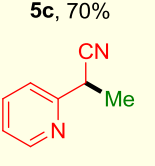

5 g, $55 \%$

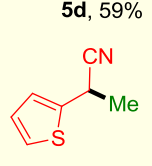

5 h, $65 \%$
${ }^{a}$ Reaction conditions: 1 (0.5 mmol), 2 (1 mmol), Mn-2 (0.005 $\mathrm{mmol}), \mathrm{Cs}_{2} \mathrm{CO}_{3}(0.05 \mathrm{mmol})$, toluene $(0.5 \mathrm{~mL}), 135{ }^{\circ} \mathrm{C}$ (aluminum block), 18 h. ${ }^{b} \mathbf{M n}-2(0.025 \mathrm{mmol})$. ${ }^{c}$ Reaction conditions: 1 (0.5 mmol), methanol $(1 \mathrm{~mL}), \mathbf{M n}-2$ (0.025 mmol), $\mathrm{Cs}_{2} \mathrm{CO}_{3}(0.05 \mathrm{mmol}), 1,4$-dioxane $(1 \mathrm{~mL}), 24 \mathrm{~h}$. 
the desired products $\mathbf{4 a - 4 f}$ in very good isolated yields. Furthermore, a naphthyl-substituted product $\mathbf{4 g}$ was obtained in $83 \%$ yield. Similar to the scope of the nitrile, alcohols bearing heterocyles (pyridine, thiophene, furane) could also be used to access the corresponding products $\mathbf{4 h}-\mathbf{4 k}$. Moreover, this protocol is not limited to benzlic alcohols, as exemplified by the rapid incorporation of different aliphatic alcohols under the same reaction conditions $(\mathbf{4 l - 4 p})$, whereby unsaturated $\mathrm{C}=\mathrm{C}$ bonds remain intact (4o).

However, a higher activation energy barrier needs to be overcome for the dehydrogenation of the methanol compared with other alcohols, our catalytic system proved to be suitable for the challenging $\alpha$-methylation of nitriles. Slightly modified reaction conditions have to be used to successfully accomplish the C1-alkylation. Indeed, a broad series of differently substituted nitriles, including heterocycles, can be methylated to deliver the products $\mathbf{5 a}-\mathbf{5 h}$ in moderate to good yields (Scheme 2C). Interestingly, our base metal catalyzed procedure shows superior catalytic activity to the recently disclosed Ru-catalyzed protocol. ${ }^{12 \mathrm{a}}$

In order to gain more insight into the reaction mechanism we monitored the reaction progress between phenylacetonitrile (1a) and 1-butanol (2a) catalyzed by Mn-2 (Figure 1). After 1 hour, we observed $83 \%$ of conversion of the nitrile 1a and the formation of $66 \%$ yield of $\mathbf{3 a}$ along with $15 \%$ of the unsaturated intermediate 3a'. After 3 hours, almost full conversion of 1a was obtained with $80 \%$ yield of the $\mathbf{3 a}$, while the amount of the remaining intermediate 3a' was still constant (ca.15\%). Complete conversion was observed after 18 hours. Subsequently, we decided to carry out several control NMR experiments (Figure 2). The treatment of Mn-2 with one equivalent of $t$ - $\mathrm{BuOK}$ at room temperature for one hour in $\mathrm{C}_{6} \mathrm{D}_{6}$ led to the formation of the soluble imido complex Mn-2a as confirmed by the ${ }^{31} \mathrm{P}-\mathrm{NMR}$ spectrum (91.02 ppm). When one equivalent of 4-flurophenylacetonitrile was added, we immediately observed the formation of two new broad peaks at 73.65 and $78.82 \mathrm{ppm}$ at ${ }^{31} \mathrm{P}-\mathrm{NMR}$, indicating the coordination between the manganese catalyst and the nitrile substrate.

Based on the experimental background and the DFT studies, a proposed reaction mechanism is shown in Scheme 3. Initially, the manganese pre-catalyst reacts with the base to generate an imido complex as the active catalytic species (Scheme 3a). This imido complex activates the alcohol via dehydrogenation with the simultaneous formation of the manganese species H-Mn-N-H. Most likely, this reaction takes place via the formation of a Mn-alkoxide intermediate. In addition to the role of the manganese catalyst in the alcohol activation, the NMR experiment indicates the role of the metal catalyst in the $\mathrm{C}-\mathrm{H}$ activation of the nitrile substrate. ${ }^{24}$ The two new broad peaks in figure $\mathbf{2 c}$ most likely correlate to the species $\mathbf{A}$ and $\mathbf{B}$ in scheme $3 \mathrm{~b}$. The initial coordination of the nitrile substrate with the $16 \mathrm{e}$ species lead to the formation of the molecule $\mathbf{A}$. Then, the ligand plays a crucial role for the hydrogen abstraction and the generation of the highly nucleophilic species $\mathbf{B} .^{25}$ Thus, the same metal catalyst simultaneously activates both of the substrates to produce the electrophile (aldehyde) and nucleophile B. the addition of the intermediate $\mathbf{B}$ to the in-situ generated aldehyde delivers the intermediate $\mathbf{C}$ and one molecule of water. This intermediate leads to the formation of the olefin intermediate 3' and regenerates the Mn-2a. Finally, the shuttled hydrogen on the $\mathrm{H}-\mathrm{Mn}-\mathrm{N}-\mathrm{H}$ is transferred to 3' to produce the $\alpha$-alkylated nitrile $\mathbf{3}$ via a metal-ligand cooperative mechanism.

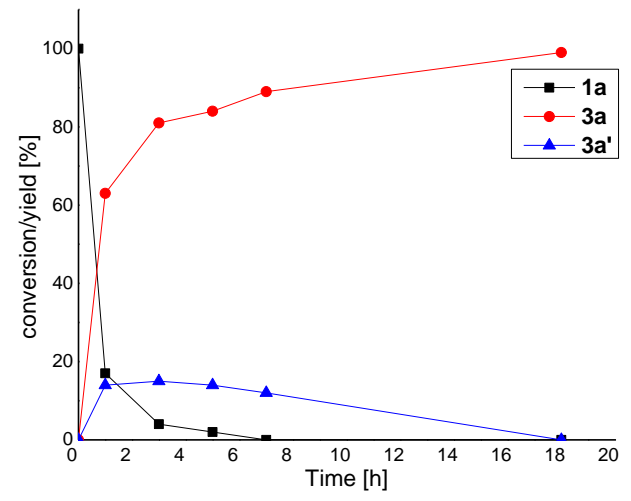

Figure 1. Monitoring of the reaction progress (reaction conditions as in table 1, entry 11).

a)

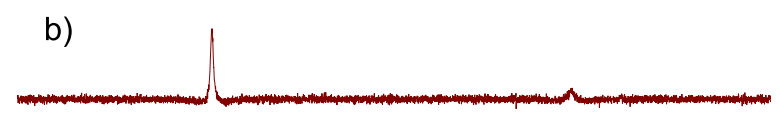

c)

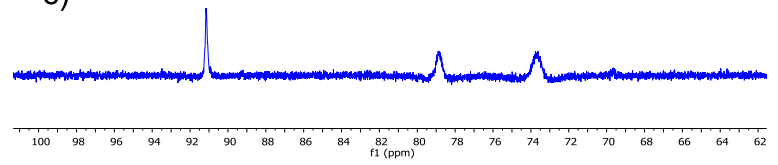

Figure 2. ${ }^{31} \mathrm{P}$ NMR spectra at RT. a) Mn-2 in $\mathrm{CD}_{2} \mathrm{Cl}_{2}$. b) Mn$\mathbf{2}+1$ equiv. $t$-BuOK in $\mathrm{C}_{6} \mathrm{D}_{6}$. c) $\mathbf{M n}-\mathbf{2}+1$ equiv. of $t$ $\mathrm{BuOK}+1$ equiv. of 4-flurophenylacetonitrile in $\mathrm{C}_{6} \mathrm{D}_{6}$.

\section{Scheme 3. Proposed Reaction Mechanism}

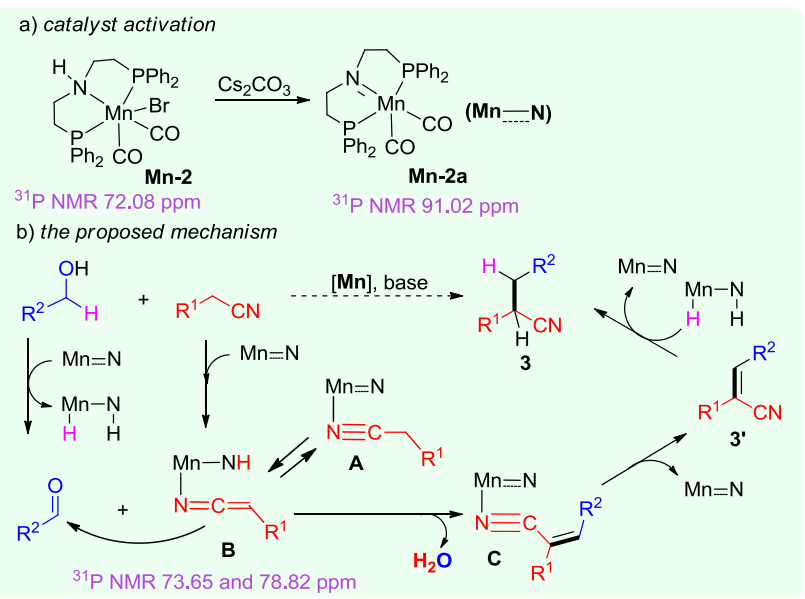

\section{CONCLUSION}

In summary, manganese PNP pincer catalyzed alkylation of nitriles with alcohols is reported. This environmentally benign, hydrogen autotransfer reaction is characterized by the absence of noble metals and stoichiometric reagents, generating water as the only side product. A broad range of alcohols could be 
used as green alkylating reagents and, importantly, the challenging methylation reaction is feasible, resulting in the desired C-methylated nitriles with excellent chemo-selectivity. The experimental studies support the reaction mechanism in which the manganese catalyst plays a multiple role in activating both the reaction partners as well as the subsequent hydrogenation of the alkenyl nitrile to selectively produce the highly value-added $\alpha$-alkylated nitriles.

\section{EXPERIMENTAL SECTION}

\section{General Method}

All reactions were carried out under an argon atmosphere using ovendried glassware. Purified compounds were further dried under high vacuum (0.01-0.05 Torr). Yields refer to purified compounds. Analytical thin-layer chromatography (TLC) was performed using silica gel 60 pre-coated aluminium plates (Macherey-Nagel $0.20 \mathrm{~mm}$ thickness) with a fluorescent indicator UV254. Visualization was performed with standard phosphomolybdic acid stain $(10 \mathrm{~g}$ in $100 \mathrm{~mL}$ EtOH) or UV light. Column chromatography was performed using Macherey-Nagel Aluminium oxide 90 neutral $(50-200 \mu \mathrm{m}) .{ }^{1} \mathrm{H}-$ NMR, ${ }^{13} \mathrm{C}-\mathrm{NMR}$ and ${ }^{31} \mathrm{P}$-NMR spectra were recorded on VNMRS400, VNMRS-600 or Mercury 300 spectrometer in $\mathrm{CDCl}_{3}$. Chemical shifts $(\delta)$ are reported in ppm and multiplicities are indicated: s (singlet), d (doublet), dd (doublet of doublet, $\mathrm{t}$ (triplet), dt (doublet of triplet), td (triplet of doublet), q, (quartet), quint (quintet) $\mathrm{m}$ (multiplet); coupling constants (J) are in Hertz (Hz). The abbreviation "vt" corresponds to a "virtual triplet" due to scalar coupling with two magnetically nonequivalent phosphorus nuclei. Mass spectra were acquired on a Finnigan SSQ7000 (EI/CI) spectrometer and high resolution mass spectra on a Finnigan MAT 95 (EI/CI) or on a ThermoFisher Scientific LTQOrbitrap XL (ESI) using ion trap as analyzer type. IR spectra were recorded on a Perkin Elmer Spectrum 100 spectrometer and are reported in terms of frequency of absorption $\left(\mathrm{cm}^{-1}\right)$.

Synthesis of tricarbonyl(2,6-bis((diphenylphosphaneyl)methyl)pyridine)manganese(I) bromide (Mn-1). ${ }^{22 \mathrm{j}}$

A flame dried Schlenk tube was charged with $\mathrm{Mn}(\mathrm{CO})_{5} \mathrm{Br}(420 \mathrm{mg}$, $1.53 \mathrm{mmol}, 1.0$ eq.) and the pincer PNP ligand (800 mg, $1.68 \mathrm{mmol}$, 1.1 eq.). The tube was evacuated and backfilled with argon for three times. THF $(25 \mathrm{~mL})$ was added and the resulting orange suspension was heated to $60{ }^{\circ} \mathrm{C}$ (oil bath) and stirred for $20 \mathrm{~h}$. The solution was allowed to cool to room temperature and THF was removed in vacuo. The work-up was done under ambient atmosphere. The yellow solid was washed three times with $n$-hexane $(3 \times 5 \mathrm{~mL})$. The crude yellow powder was taken into dichloromethane and transferred into a $50 \mathrm{~mL}$ round bottom flask to remove insoluble inorganic side products. The solution was concentrated under reduced pressure and the complex Mn-1 was isolated as a yellow powder (1.02 g, 95\%); ${ }^{1} \mathrm{H}$ NMR (300 $\left.\mathrm{MHz}, \mathrm{CD}_{2} \mathrm{Cl}_{2}\right) \delta 8.09(\mathrm{~s}, 2 \mathrm{H}), 7.81-7.26(\mathrm{~m}, 21 \mathrm{H}), 4.65(\mathrm{~s}, 4 \mathrm{H})$; ${ }^{31} \mathrm{P}\left\{{ }^{1} \mathrm{H}\right\}$ NMR (243 MHz, $\left.\mathrm{CD}_{2} \mathrm{Cl}_{2}\right) \delta$ 69.4. (s, 2P); IR (ATR) 1918, 1840, 1571, 1435, 1284, 1172, 1097, 964, 833, $696 \mathrm{~cm}^{-1}$; HRMS (ESI+): $\mathrm{m} / \mathrm{z} \cdot \mathrm{M}-\mathrm{Br}]^{+}$calcd for $\mathrm{C}_{34} \mathrm{H}_{27} \mathrm{MnNO}_{3} \mathrm{P}_{2}, 614.0841$; found, 614.0853 .

Synthesis of bromodicarbonyl(bis(2-(diphenylphosphaneyl)ethyl)amine)manganese(I) (Mn-2). ${ }^{22 \mathrm{~K}}$

A flame dried Schlenk tube was charged with the $\mathrm{HCl}$ salt of the PNP pincer ligand (478 mg, $1.0 \mathrm{mmol}, 1.0$ equiv.), toluene $(8 \mathrm{~mL})$, water $(2 \mathrm{~mL})$ and $\mathrm{NaOH}(120 \mathrm{mg}, 3.0 \mathrm{mmol}, 3.0$ equiv.). The reaction mixture was stirred at $45{ }^{\circ} \mathrm{C}$ (oil bath) for $30 \mathrm{~min}$. The two phases were separated, and the organic layer was washed with water $(5 \times 5$ $\mathrm{mL}$ ). The $\mathrm{pH}$-value was checked until the solution turned neutral. The organic layer was concentrated under reduced pressure. Subsequently, toluene $(12 \mathrm{~mL})$ and $\mathrm{Mn}(\mathrm{CO})_{5} \mathrm{Br}$ precursor were added to the Schlenk tube. The reaction mixture was heated up to $110{ }^{\circ} \mathrm{C}$ (oil bath) and the atmosphere was exchanged three times by evacuating and backfilled with argon. After the mixture was stirred for $20 \mathrm{~h}$ at reflux temperature, it was cooled to room temperature and concentrated in vacuo. The crude precipitate was washed with pentane and extracted with dichloromethane/diethylether to remove insoluble inorganic side products. The solution was concentrated under reduced pressure and dried to afford the complex Mn-2 as a yellow powder (410 mg, 65\%). ${ }^{1} \mathrm{H}$ NMR $\left(600 \mathrm{MHz}, \mathrm{CD}_{2} \mathrm{Cl}_{2}\right) \delta 7.92-7.89\left(\mathrm{~m}, 4 \mathrm{H}, \mathrm{CH}_{\mathrm{Ar}}\right), 7.60-$ $7.57\left(\mathrm{~m}, 4 \mathrm{H}, \mathrm{C} H_{\mathrm{Ar}}\right), 7.43-7.29\left(\mathrm{~m}, 12 \mathrm{H}, \mathrm{CH}_{\mathrm{Ar}}\right), 3.74-3.59(\mathrm{~m}, 2 \mathrm{H}$, $\left.\mathrm{NCH}_{2} \mathrm{CH}_{2}\right), 3.52$ (br, $\left.1 \mathrm{H}, \mathrm{NH}\right), 3.32-3.23\left(\mathrm{~m}, 2 \mathrm{H}, \mathrm{NCH}_{2} \mathrm{CH}_{2}\right), 2.78$ $-2.73\left(\mathrm{~m}, 2 \mathrm{H}, \mathrm{NCH}_{2} \mathrm{CH}_{2}\right), 2.44-2.40\left(\mathrm{~m}, 2 \mathrm{H}, \mathrm{NCH}_{2} \mathrm{CH}_{2}\right) ;{ }^{31} \mathrm{P}\left\{{ }^{1} \mathrm{H}\right\}$ NMR $\left(243 \mathrm{MHz}, \mathrm{CD}_{2} \mathrm{Cl}_{2}\right) \delta=69.7(\mathrm{~s}) ;{ }^{13} \mathrm{C}\left\{{ }^{1} \mathrm{H}\right\}$ NMR $(151 \mathrm{MHz}$, $\left.\mathrm{CD}_{2} \mathrm{Cl}_{2}\right) \delta=231.6(\mathrm{br}, \mathrm{CO}), 226.0(\mathrm{br}, \mathrm{CO}), 137.9$ (vt, $J=19.1 \mathrm{~Hz}$ $\mathrm{P}_{\text {Ar,ipso }}$ ), 135.5 (vt, $J=19.1 \mathrm{~Hz}, \mathrm{P} C_{\mathrm{Ar}, \text { ipso }}$ ), 133.8 (vt, $J=5.0 \mathrm{~Hz}$, $\left.C \mathrm{H}_{\mathrm{Ar}}\right), 130.6$ (vt, $\left.J=5.0 \mathrm{~Hz}, C \mathrm{H}_{\mathrm{Ar}}\right), 130.3\left(\mathrm{~s}, C \mathrm{H}_{\mathrm{Ar}}\right), 129.6\left(\mathrm{~s}, C \mathrm{H}_{\mathrm{Ar}}\right)$, 129.0 (vt, $J=4.3 \mathrm{~Hz}, C \mathrm{H}_{\mathrm{Ar}}$ ), 128.7 (vt, $J=4.6 \mathrm{~Hz}, C \mathrm{H}_{\mathrm{Ar}}$ ), 53.0 (vt, $J$ $=4.7 \mathrm{~Hz}, \mathrm{NCH}_{2} \mathrm{CH}_{2}$ ), 28.4 (vt, $J=8.9 \mathrm{~Hz}, \mathrm{NCH}_{2} \mathrm{CH}_{2}$ ); IR (ATR): $v^{-1}$ $3189,1910,1826 \mathrm{~cm}^{-1}$; HRMS (ESI+): $\mathrm{m} / z[\mathrm{M}-\mathrm{Br}]^{+}$: calcd for $\mathrm{C}_{30} \mathrm{H}_{29} \mathrm{MnNO}_{2} \mathrm{P}_{2}$, 552.1049; found, 552.1050.

Synthesis of dicarbonyl(bis(2-(diphenylphosphaneyl)ethyl)amide)manganese(I) (Mn-2a).

In an argon filled glovebox complex Mn-2 (127 mg, $0.2 \mathrm{mmol})$ was introduced into a $25 \mathrm{~mL}$ Schlenk tube and was dissolved in toluene (5 $\mathrm{mL})$. After $2 \mathrm{~min}$ stirring, $t$-BuOK $(34 \mathrm{mg}, 0.3 \mathrm{mmol})$ was added upon which the yellow solution turned deep red. The reaction mixture was stirred for $1 \mathrm{~h}$ at $50{ }^{\circ} \mathrm{C}$ (oil bath). Then the mixture was filtered and the solution was concentrated under reduced pressure to give a deep red powder $(75 \mathrm{mg}, 68 \%)$ that was stored in glovebox. ${ }^{1} \mathrm{H}$ NMR $\left(600 \mathrm{MHz}, \mathrm{C}_{6} \mathrm{D}_{6}\right) \delta 7.73-7.71\left(\mathrm{~m}, 8 \mathrm{H}, \mathrm{CH}_{\mathrm{Ar}}\right), 7.10-7.08(\mathrm{~m}, 8 \mathrm{H}$, $\left.\mathrm{CH}_{\mathrm{Ar}}\right), 7.04-7.02(\mathrm{~m}, 4 \mathrm{H}), 3.25-3.24\left(\mathrm{~m}, 4 \mathrm{H}, \mathrm{NCH}_{2} \mathrm{CH}_{2}\right), 2.35-$ $2.32\left(\mathrm{~m}, 4 \mathrm{H}, \mathrm{NCH}_{2} \mathrm{CH}_{2}\right) ;{ }^{31} \mathrm{P}\left\{{ }^{1} \mathrm{H}\right\}$ NMR $\left(243 \mathrm{MHz}, \mathrm{C}_{6} \mathrm{D}_{6}\right) \delta 91.06(\mathrm{~s})$; ${ }^{13} \mathrm{C}\left\{{ }^{1} \mathrm{H}\right\}$ NMR (151 MHz, $\left.\mathrm{C}_{6} \mathrm{D}_{6}\right) \delta=233.5$ (br, CO), 136.6 (vt, $J=$ $\left.18.0 \mathrm{~Hz}, \mathrm{P} C_{\mathrm{Ar}, \mathrm{ipso}}\right), 132.7\left(\mathrm{~s}, C \mathrm{H}_{\mathrm{Ar}}\right), 129.8\left(\mathrm{~s}, C \mathrm{H}_{\mathrm{Ar}}\right), 128.8(\mathrm{vt}, J=4.3$ $\left.\mathrm{Hz}, \mathrm{CH}_{\mathrm{Ar}}\right), 62.1\left(\mathrm{NCH}_{2} \mathrm{CH}_{2}\right), 33.7\left(\mathrm{NCH}_{2} \mathrm{CH}_{2}\right)$; IR (ATR): 1903, $1828 \mathrm{~cm}^{-1}$, (no $v_{\mathrm{NH}}$ band observed).

Synthesis of tricarbonyl(2-(diphenylphosphaneyl)-N-(pyridin-2ylmethyl)ethan-1-amine)manganese(I) bromide (Mn-3). ${ }^{206}$

A flame dried Schlenk tube was charged with the PNN pincer ligand (300 mg, $1.07 \mathrm{mmol}, 1$ eq.) and $\mathrm{Mn}(\mathrm{CO})_{5} \mathrm{Br}(293 \mathrm{mg}, 1.07 \mathrm{mmol}, 1.0$ eq.). The Schlenk tube was evacuated and backfilled with argon for several time. Afterwards, $15 \mathrm{~mL}$ of degassed THF was added and the reaction mixture was stirred at $80{ }^{\circ} \mathrm{C}$ (oil bath) for $20 \mathrm{~h}$. The suspension was allowed to cool to room temperature and the yellow precipitate was filtered off and washed with diethyl ether and n-hexane. The remaining solid was dried under vacuum to afford the complex Mn-3 as a yellow powder $(0.49 \mathrm{~g}, 84 \%) ;{ }^{1} \mathrm{H}$ NMR $(600 \mathrm{MHz}, \mathrm{DMSO}-d 6): \delta$ $7.93-7.92(\mathrm{~m}, 1 \mathrm{H}), 7.87-7.84(\mathrm{~m}, 2 \mathrm{H}), 7.81-7.78(\mathrm{~m}, 1 \mathrm{H}), 7.58$ $7.49(\mathrm{~m}, 4 \mathrm{H}), 7.39-7.37(\mathrm{~m}, 1 \mathrm{H}), 7.31-7.29(\mathrm{~m}, 2 \mathrm{H}), 7.20-7.09$ (m, 2H), $6.94-6.92(\mathrm{~m}, 1 \mathrm{H}), 4.58-4.54(\mathrm{~m}, 1 \mathrm{H}), 4.38(\mathrm{~m}, 1 \mathrm{H}), 3.28$ $-3.20(\mathrm{~m}, 1 \mathrm{H}), 3.04-3.00(\mathrm{~m}, 1 \mathrm{H}), 2.95-2.89(\mathrm{~m}, 1 \mathrm{H}), 2.40-2.27$ $(\mathrm{m}, 1 \mathrm{H}) ;{ }^{13} \mathrm{C}\left\{{ }^{1} \mathrm{H}\right\}$ NMR (151 MHz, DMSO-d6) $\delta 221.2,219.9,215.3$, $162.1,153.0,139.2,132.4,132.0,131.1,131.0,130.9,130.8,129.9$, $129.8,129.6,125.0,122.4,59.8,53.8(\mathrm{~d}, J=8.8 \mathrm{~Hz}), 22.7(\mathrm{~d}, J=22.7$ $\mathrm{Hz}) ;{ }^{31} \mathrm{P}\left\{{ }^{1} \mathrm{H}\right\}$ NMR (242 MHz, DMSO- $d 6$ ) $\delta 65.86$; IR (ATR): 3045 , 2891, 2024, 1914, 1843, 1477, 1434, 1099, 892, 752, $693 \mathrm{~cm}^{-1 ;}$ HRMS (ESI): $\mathrm{m} / \mathrm{z},[\mathrm{M}-\mathrm{Br}]^{+}$calcd for $\mathrm{C}_{23} \mathrm{H}_{21} \mathrm{MnN}_{2} \mathrm{O}_{3} \mathrm{P}, 459.0665$; found, 459.0675 .

General procedure of $\boldsymbol{C}$-alkylation of nitriles using aliphatic and benzylic alcohols

A glass pressure tube $(10 \mathrm{~mL})$ equipped with a magnetic stirrer was charged with Mn-2 (3.2 mg, $0.005 \mathrm{mmol}), \mathrm{Cs}_{2} \mathrm{CO}_{3}(16.3 \mathrm{mg}, 0.05$ $\mathrm{mmol})$. A rubber septum was attached to the tube and the reaction vessel was evacuated and backfilled with argon for three times. Under an inert atmosphere primary alcohol $(1.0 \mathrm{mmol})$, nitrile $(0.5 \mathrm{mmol})$ and toluene $(0.5 \mathrm{~mL})$ were added and the tube was closed with a screw cap. The resulting mixture was stirred at $135{ }^{\circ} \mathrm{C}$ (aluminum block) for $18 \mathrm{~h}$ under argon atmosphere. Upon cooling down to room temperature the residue was directly purified by flash column chromatography on silica gel eluting with pentane: diethyl ether $(20: 1(\mathrm{v} / \mathrm{v}))$ to give the pure alkylated nitrile.

2-phenylhexanenitrile (3a). ${ }^{12 \mathrm{a}}$ Colorless oil; $78 \mathrm{mg}(88 \%){ }^{1} \mathrm{H}$ NMR $\left(400 \mathrm{MHz}, \mathrm{CDCl}_{3}\right) \delta 7.46-7.27(\mathrm{~m}, 5 \mathrm{H}), 3.77(\mathrm{dd}, J=8.5,6.3 \mathrm{~Hz}$, 
1H), $2.04-1.74(\mathrm{~m}, 2 \mathrm{H}), 1.60-1.25(\mathrm{~m}, 4 \mathrm{H}), 0.92(\mathrm{t}, J=7.2 \mathrm{~Hz}$, $3 \mathrm{H}) ;{ }^{13} \mathrm{C}\left\{{ }^{1} \mathrm{H}\right\} \mathrm{NMR}\left(101 \mathrm{MHz}, \mathrm{CDCl}_{3}\right) \delta 136.2,129.1,128.0,127.3$, 121.0, 37.5, 35.7, 29.2, 22.2, 13.8.

2-(p-tolyl)hexanenitrile $(3 \boldsymbol{b})^{13 \mathrm{~d}}$ Colorless oil; $81 \mathrm{mg}(87 \%) ;{ }^{1} \mathrm{H} \mathrm{NMR}$ $\left(600 \mathrm{MHz}, \mathrm{CDCl}_{3}\right) \delta 7.25-7.16(\mathrm{~m}, 4 \mathrm{H}), 3.74(\mathrm{dd}, J=8.6,6.3 \mathrm{~Hz}$, $1 \mathrm{H}), 2.36(\mathrm{~s}, 3 \mathrm{H}), 1.99-1.77(\mathrm{~m}, 2 \mathrm{H}), 1.55-1.29(\mathrm{~m}, 4 \mathrm{H}), 0.92(\mathrm{t}, J$ $=7.3 \mathrm{~Hz}, 3 \mathrm{H}) ;{ }^{13} \mathrm{C}\left\{{ }^{1} \mathrm{H}\right\}$ NMR $\left(151 \mathrm{MHz}, \mathrm{CDCl}_{3}\right) \delta$ 137.8, 133.1, $129.7,127.2,121.2,37.0,35.7,29.2,22.2,21.1,13.9$.

2-(2-methoxyphenyl)hexanenitrile (3c). ${ }^{12 \mathrm{a}}$ Colorless oil; $96 \mathrm{mg}(94 \%)$; eluent mixture: pentane: diethyl ether (10:1 (v/v)), ${ }^{1} \mathrm{H}$ NMR (400 $\left.\mathrm{MHz}, \mathrm{CDCl}_{3}\right) \delta 7.42-7.40(\mathrm{~m}, 1 \mathrm{H}), 7.30-7.27(\mathrm{~m}, 1 \mathrm{H}), 7.00-6.96$ (m, 1H), $6.91-6.82(\mathrm{~m}, 1 \mathrm{H}), 4.19(\mathrm{dd}, J=8.2,6.3 \mathrm{~Hz}, 1 \mathrm{H}), 3.85(\mathrm{~s}$, $3 \mathrm{H}), 1.86(\mathrm{~m}, 2 \mathrm{H}), 1.62-1.23(\mathrm{~m}, 4 \mathrm{H}), 0.92(\mathrm{t}, J=7.2 \mathrm{~Hz}, 3 \mathrm{H})$; ${ }^{13} \mathrm{C}\left\{{ }^{1} \mathrm{H}\right\}$ NMR $\left(101 \mathrm{MHz}, \mathrm{CDCl}_{3}\right) \delta$ 156.1, 129.3, 128.3, 124.5, $121.4,120.9,110.8,55.5,33.5,31.4,29.4,22.1,13.8$.

2-(3,4-dimethoxyphenyl)hexanenitrile (3d). Colorless oil; $103 \mathrm{mg}$ (88\%); eluent mixture: pentane: diethyl ether $(5: 1(\mathrm{v} / \mathrm{v})) ;{ }^{1} \mathrm{H}$ NMR $\left(600 \mathrm{MHz}, \mathrm{CDCl}_{3}\right) \delta 6.85-6.76(\mathrm{~m}, 3 \mathrm{H}), 3.86(\mathrm{~s}, 3 \mathrm{H}), 3.84(\mathrm{~s}, 3 \mathrm{H})$, $3.68(\mathrm{dd}, J=8.7,6.3 \mathrm{~Hz}, 1 \mathrm{H}), 1.94-1.75(\mathrm{~m}, 2 \mathrm{H}), 1.50-1.26(\mathrm{~m}$, $4 \mathrm{H}), 0.87(\mathrm{t}, J=7.3 \mathrm{~Hz}, 3 \mathrm{H}) ;{ }^{13} \mathrm{C}\left\{{ }^{1} \mathrm{H}\right\} \mathrm{NMR}\left(151 \mathrm{MHz}, \mathrm{CDCl}_{3}\right) \delta$ $149.3,148.7,128.4,121.2,119.5,111.3,110.2,110.1,56.0,55.9$, 36.9, 35.6, 29.1, 22.1, 13.8; IR: 2936, 2865, 2087, 1906, 1595, 1457, 1343, 1253, 1146, 1026, 913, 809, $759 \mathrm{~cm}^{-1}$. HRMS (ESI+): $\mathrm{m} / \mathrm{z}[\mathrm{M}]^{+}$ calcd for $\mathrm{C}_{14} \mathrm{H}_{19} \mathrm{O}_{2} \mathrm{~N}, 233.1410$; found 233.1413.

2-(benzo[d][1,3]dioxol-5-yl)hexanenitrile (3e). ${ }^{12 \mathrm{a}}$ Colorless oil; 87 $\mathrm{mg}(80 \%)$; eluent mixture: pentane: diethyl ether (5:1 (v/v)); ${ }^{1} \mathrm{H}$ NMR $\left(600 \mathrm{MHz}, \mathrm{CDCl}_{3}\right) \delta 6.81-6.73(\mathrm{~m}, 3 \mathrm{H}), 5.96(\mathrm{~s}, 2 \mathrm{H}), 3.67(\mathrm{dd}, J=$ 8.5, $6.4 \mathrm{~Hz}, 1 \mathrm{H}), 1.93-1.74(\mathrm{~m}, 2 \mathrm{H}), 1.50-1.27(\mathrm{~m}, 4 \mathrm{H}), 0.94-$ $0.85(\mathrm{~m}, 3 \mathrm{H}) ;{ }^{13} \mathrm{C}\left\{{ }^{1} \mathrm{H}\right\}$ NMR $\left(151 \mathrm{MHz}, \mathrm{CDCl}_{3}\right) \delta$ 148.2, 147.4, 129.8, 121.1, 120.7, 108.6, 107.7, 107.7, 101.4, 37.1, 35.7, 29.1, 22.1, 13.8 .

2-(4-chlorophenyl)hexanenitrile (3f). ${ }^{26 \mathrm{a}}$ Colorless oil; $82 \mathrm{mg}(79 \%)$; ${ }^{1} \mathrm{H}$ NMR $\left(600 \mathrm{MHz}, \mathrm{CDCl}_{3}\right) \delta 7.35-7.25(\mathrm{~m}, 4 \mathrm{H}), 3.75(\mathrm{dd}, J=8.6$, $6.2 \mathrm{~Hz}, 1 \mathrm{H}), 1.97-1.77(\mathrm{~m}, 2 \mathrm{H}), 1.51-1.28(\mathrm{~m}, 4 \mathrm{H}), 0.90(\mathrm{t}, J=7.3$ $\mathrm{Hz}, 3 \mathrm{H}) ;{ }^{13} \mathrm{C}\left\{{ }^{1} \mathrm{H}\right\}$ NMR $\left(151 \mathrm{MHz}, \mathrm{CDCl}_{3}\right) \delta$ 134.6, 134.0, 129.3, 128.7, 120.6, 36.9, 35.6, 29.1, 22.1, 13.8 .

2-(2-chlorophenyl)hexanenitrile (3g). Colorless oil; $82 \mathrm{mg}(79 \%) ;{ }^{1} \mathrm{H}$ $\operatorname{NMR}\left(600 \mathrm{MHz}, \mathrm{CDCl}_{3}\right) \delta 7.56-7.55(\mathrm{~m}, 1 \mathrm{H}), 7.42-7.36(\mathrm{~m}, 1 \mathrm{H})$, $7.32-7,31(\mathrm{~m}, 1 \mathrm{H}), 7.28-7.25(\mathrm{~m}, 1 \mathrm{H}), 4.28(\mathrm{dd}, J=9.0,5.6 \mathrm{~Hz}$, $1 \mathrm{H}), 1.96-1.79(\mathrm{~m}, 2 \mathrm{H}), 1.61-1.30(\mathrm{~m}, 4 \mathrm{H}), 0.92(\mathrm{t}, J=7.4 \mathrm{~Hz}$ $3 \mathrm{H}) .{ }^{13} \mathrm{C}\left\{{ }^{1} \mathrm{H}\right\} \mathrm{NMR}\left(151 \mathrm{MHz}, \mathrm{CDCl}_{3}\right) \delta 134.0,132.6,130.0,129.5$, $128.9,127.7,120.5,34.7,34.0,29.3,22.1,13.9$; IR: 3424, 2935 2866, 2666, 2323, 2103, 1921, 1728, 1466, 1278, 1040, $754 \mathrm{~cm}^{-1}$; HRMS (ESI+): $m / z[\mathrm{M}+\mathrm{Na}]^{+}$calcd for $\mathrm{C}_{12} \mathrm{H}_{14} \mathrm{~N}_{1} \mathrm{Cl}_{1} \mathrm{Na}_{1} 230.0707$; found, 230.0707 .

4-(1-cyanopentyl)benzonitrile (3h). Yellowish oil; $52 \mathrm{mg}(53 \%) ;{ }^{1} \mathrm{H}$ $\operatorname{NMR}(600 \mathrm{MHz}, \mathrm{CDCl} 3) \delta 7.70-7.68(\mathrm{~m}, 2 \mathrm{H}), 7.47-7.46(\mathrm{~m}, 2 \mathrm{H})$, $3.85(\mathrm{dd}, J=8.6,6.1 \mathrm{~Hz}, 1 \mathrm{H}), 1.98-1.82(\mathrm{~m}, 2 \mathrm{H}), 1.54-1.29(\mathrm{~m}$, $4 \mathrm{H}), 0.91(\mathrm{t}, J=7.3 \mathrm{~Hz}, 3 \mathrm{H}) ;{ }^{13} \mathrm{C}\left\{{ }^{1} \mathrm{H}\right\}$ NMR $\left(151 \mathrm{MHz}, \mathrm{CDCl}_{3}\right) \delta$ 141.3, 133.0, 128.2, 119.8, 118.3, 112.4, 37.6, 35.5, 29.1, 22.2, 13.9; IR: $3470,3064,2958,2867,2231,1610,1506,1462,1414,1381$, 1115, 1021, $841 \mathrm{~cm}^{-1}$; HRMS (ESI+): $\mathrm{m} / z[\mathrm{M}+\mathrm{H}]^{+}$calcd for $\mathrm{C}_{13} \mathrm{H}_{15} \mathrm{~N}_{2} 199.1230$; found 199.1230 .

2-(pyridin-2-yl)hexanenitrile (3i). Colorless oil; $86 \mathrm{mg}$ (99\%); eluent mixture: pentane: diethyl ether (10:1 (v/v)); ${ }^{1} \mathrm{H}$ NMR $(600 \mathrm{MHz}$, $\left.\mathrm{CDCl}_{3}\right) \delta 8.58-8.51(\mathrm{~m}, 1 \mathrm{H}), 7.70-7.67(\mathrm{~m}, 1 \mathrm{H}), 7.42-7.37(\mathrm{~m}$ $1 \mathrm{H}), 7.22-7.20(\mathrm{~m}, 1 \mathrm{H}), 3.94(\mathrm{dd}, J=8.3,6.4 \mathrm{~Hz}, 1 \mathrm{H}), 1.99-1.95$ (m, 2H), $1.53-1.25(\mathrm{~m}, 4 \mathrm{H}), 0.86(\mathrm{t}, J=7.4 \mathrm{~Hz}, 3 \mathrm{H}) ;{ }^{13} \mathrm{C}\left\{{ }^{1} \mathrm{H}\right\} \mathrm{NMR}$ $\left(151 \mathrm{MHz}, \mathrm{CDCl}_{3}\right) \delta 155.4,149.9,137.3,122.9,121.7,120.2,39.9$, 33.9, 29.1, 22.1, 13.8; IR: 3180, 2933, 2867, 2322, 1920, 1738, 1586, 1467, 1437, 1301, 1206, 994, 755, $665 \mathrm{~cm}^{-1}$; HRMS (ESI+): $\mathrm{m} / \mathrm{z}[\mathrm{M}]^{+}$ calcd for $\mathrm{C}_{11} \mathrm{H}_{14} \mathrm{~N}_{2}$, 174.1152; found 174.1145.

2-(thiophen-2-yl)hexanenitrile (3j). Yellowish oil; $58 \mathrm{mg}(65 \%),{ }^{1} \mathrm{H}$ NMR $(600 \mathrm{MHz}, \mathrm{CDCl} 3) \delta 7.27-7.26(\mathrm{~m}, 1 \mathrm{H}), 7.06-7.05(\mathrm{~d}, J=$ $3.5 \mathrm{~Hz}, 1 \mathrm{H}), 6.98-6.97(\mathrm{~m}, 1 \mathrm{H}), 4.06(\mathrm{dd}, J=8.4,6.3 \mathrm{~Hz}, 1 \mathrm{H}), 2.06$ $-1.91(\mathrm{~m}, 2 \mathrm{H}), 1.58-1.43(\mathrm{~m}, 2 \mathrm{H}), 1.43-1.33(\mathrm{~m}, 2 \mathrm{H}), 0.93(\mathrm{t}, J=$ $7.3 \mathrm{~Hz}, 3 \mathrm{H}) ;{ }^{13} \mathrm{C}\left\{{ }^{1} \mathrm{H}\right\}$ NMR $\left(151 \mathrm{MHz}, \mathrm{CDCl}_{3}\right) \delta 138.2,127.1,126.1$, 125.5, 120.1, 35.7, 32.6, 29.1, 22.1, 13.9; IR: 3468, 3109, 2957, 2865,
2242, 1728, 1461, 1379, 1238, 1038, 836, $703 \mathrm{~cm}^{-1}$; HRMS (ESI+): $m / z[\mathrm{M}+\mathrm{Na}]^{+}$calcd for $\mathrm{C}_{10} \mathrm{H}_{13} \mathrm{~N}_{1} \mathrm{NaS}, 202.0661$; found 202.0659 .

2,3-diphenylpropanenitrile (4a). ${ }^{10}$ Colorless oil; $89 \mathrm{mg}(86 \%) ;{ }^{1} \mathrm{H}$ NMR $\left(600 \mathrm{MHz}, \mathrm{CDCl}_{3}\right) \delta 7.41-7.26(\mathrm{~m}, 8 \mathrm{H}), 7.18-7.14(\mathrm{~m}, 2 \mathrm{H})$ $4.02(\mathrm{dd}, J=8.5,6.4 \mathrm{~Hz}, 1 \mathrm{H}), 3.21(\mathrm{dd}, J=13.6,8.4 \mathrm{~Hz}, 1 \mathrm{H}), 3.15$ $(\mathrm{dd}, J=13.7,6.4 \mathrm{~Hz}, 1 \mathrm{H}) ;{ }^{13} \mathrm{C}\left\{{ }^{1} \mathrm{H}\right\} \mathrm{NMR}\left(151 \mathrm{MHz}, \mathrm{CDCl}_{3}\right) \delta 136.4$, 135.3, 129.3, 129.1, 128.7, 128.3, 127.6, 127.5, 120.5, 42.3, 39.9.

3-phenyl-2-(pyridin-2-yl)propanenitrile $(\mathbf{4 b}) .{ }^{13 \mathrm{c}}$ Yellowish oil; $76 \mathrm{mg}$ (73\%); eluent mixture: pentane: diethyl ether $(10: 1(\mathrm{v} / \mathrm{v})) ;{ }^{1} \mathrm{H}$ NMR $\left(600 \mathrm{MHz}, \mathrm{CDCl}_{3}\right) \delta 8.65-8.64(\mathrm{~m}, 1 \mathrm{H}), 7.68-7.65(\mathrm{~m}, 1 \mathrm{H}), 7.30-$ $7.24(\mathrm{~m}, 5 \mathrm{H}), 7.18-7.16(\mathrm{~m}, 2 \mathrm{H}), 4.21(\mathrm{dd}, J=8.7,5.9 \mathrm{~Hz}, 1 \mathrm{H}), 3.37$ (dd, $J=13.6,5.9 \mathrm{~Hz}, 1 \mathrm{H}), 3.26(\mathrm{dd}, J=13.6,8.7 \mathrm{~Hz}, 1 \mathrm{H}) ;{ }^{13} \mathrm{C}\left\{{ }^{1} \mathrm{H}\right\}$ NMR $\left(151 \mathrm{MHz}, \mathrm{CDCl}_{3}\right) \delta 154.6,150.1,137.3,136.4,129.3,128.8$, $127.5,123.2,122.3,119.8,42.2,40.2$.

2-phenyl-3-(p-tolyl)propanenitrile $(\mathbf{4 c}) .{ }^{14}$ White solid; $101 \mathrm{mg}(91 \%)$; ${ }^{1} \mathrm{H}$ NMR $\left(600 \mathrm{MHz}, \mathrm{CDCl}_{3}\right) \delta 7.39-7.33(\mathrm{~m}, 3 \mathrm{H}), 7.29-7.28(\mathrm{~m}$, $2 \mathrm{H}), 7.13-7.11(\mathrm{~m}, 2 \mathrm{H}), 7.06-7.04(\mathrm{~m}, 2 \mathrm{H}), 3.99(\mathrm{dd}, J=8.5,6.3$ $\mathrm{Hz}, 1 \mathrm{H}), 3.16(\mathrm{dd}, J=13.7,8.5 \mathrm{~Hz}, 1 \mathrm{H}), 3.11(\mathrm{dd}, J=13.7,6.4 \mathrm{~Hz}$, $1 \mathrm{H}), 2.34(\mathrm{~s}, 3 \mathrm{H}) ;{ }^{13} \mathrm{C}\left\{{ }^{1} \mathrm{H}\right\}$ NMR $\left(151 \mathrm{MHz}, \mathrm{CDCl}_{3}\right) \delta$ 137.1, 135.4, 133.3, 129.4, 129.2, 129.1, 128.2, 127.6, 120.6, 41.9, 40.0, 21.2.

3-(3,4-dimethoxyphenyl)-2-phenylpropanenitrile $\quad(\mathbf{4 d}){ }^{13 \mathrm{c}}$ Off-white solid; $121 \mathrm{mg}$ (91\%); eluent mixture: pentane: diethyl ether (10:1 $(\mathrm{v} / \mathrm{v})) ;{ }^{1} \mathrm{H}$ NMR $\left(600 \mathrm{MHz}, \mathrm{CDCl}_{3}\right) \delta 7.38-7.30(\mathrm{~m}, 3 \mathrm{H}), 7.26-$ $7.22(\mathrm{~m}, 2 \mathrm{H}), 6.78-6.79(\mathrm{~m}, 1 \mathrm{H}), 6.71-6.69(\mathrm{~m}, 1 \mathrm{H}), 6.52-6.51$ $(\mathrm{m}, 1 \mathrm{H}), 3.98(\mathrm{dd}, J=7.8,6.4 \mathrm{~Hz}, 1 \mathrm{H}), 3.86(\mathrm{~s}, 3 \mathrm{H}), 3.76(\mathrm{~s}, 3 \mathrm{H})$, $3.14(\mathrm{dd}, J=13.7,7.9 \mathrm{~Hz}, 1 \mathrm{H}), 3.09(\mathrm{dd}, J=13.7,6.4 \mathrm{~Hz}, 1 \mathrm{H})$; ${ }^{13} \mathrm{C}\left\{{ }^{1} \mathrm{H}\right\}$ NMR $\left(151 \mathrm{MHz}^{\left.-\mathrm{CDCl}_{3}\right) \delta} 148.8,148.4,135.3,129.1\right.$, 128.7, 128.3, 127.7, 121.5, 120.6, 112.5, 111.2, 56.0, 55.9, 41.9, 40.0.

3-(benzo[d][1,3]dioxol-4-yl)-2-phenylpropanenitrile (4e). Colorless oil; $91 \mathrm{mg}$ (72\%); eluent mixture: pentane: diethyl ether $(5: 1(\mathrm{v} / \mathrm{v}))$; ${ }^{1} \mathrm{H}$ NMR $\left(600 \mathrm{MHz}, \mathrm{CDCl}_{3}\right) \delta 7.39-7.30(\mathrm{~m}, 3 \mathrm{H}), 7.28-7.25(\mathrm{~m}$, $2 \mathrm{H}), 6.73-6.72(\mathrm{~m}, 1 \mathrm{H}), 6.63-6.58(\mathrm{~m}, 2 \mathrm{H}), 5.94(\mathrm{~s}, 2 \mathrm{H}), 3.96(\mathrm{dd}$, $J=8.4,6.4 \mathrm{~Hz}, 1 \mathrm{H}), 3.10(\mathrm{dd}, J=13.7,8.3 \mathrm{~Hz}, 1 \mathrm{H}), 3.05(\mathrm{dd}, J=$ $13.8,6.5 \mathrm{~Hz}, 1 \mathrm{H}) ;{ }^{13} \mathrm{C}\left\{{ }^{1} \mathrm{H}\right\} \mathrm{NMR}\left(151 \mathrm{MHz}, \mathrm{CDCl}_{3}\right) \delta 147.8,147.0$, $135.2,130.0,129.1,128.3,127.6,122.6,120.5,109.6,108.5,101.2$, 42.0, 40.1; IR: 3064, 3031, 2899, 2241, 1685, 1605, 1495, 1446, $1249,1194,1100,1039,810,751,699 \mathrm{~cm}^{-1}$; HRMS (ESI+): $\mathrm{m} / z[\mathrm{M}+$ $\mathrm{H}]^{+}$calcd for $\mathrm{C}_{16} \mathrm{H}_{14} \mathrm{O}_{2} \mathrm{~N}, 252.1019$; found 252.1017 .

2-phenyl-3-(3-(trifluoromethyl)phenyl)propanenitrile (4f). Colorless oil; $105 \mathrm{mg}$ (76\%); ${ }^{1} \mathrm{H}$ NMR $\left(600 \mathrm{MHz}, \mathrm{CDCl}_{3}\right) \delta 7.55-7.53(\mathrm{~m}$, $1 \mathrm{H}), 7.44-7.42(\mathrm{~m}, 1 \mathrm{H}), 7.39-7.33(\mathrm{~m}, 4 \mathrm{H}), 7.30-7.29(\mathrm{~m}, 1 \mathrm{H})$, $7.26-7.23(\mathrm{~m}, 2 \mathrm{H}), 4.04(\mathrm{dd}, J=8.1,6.5 \mathrm{~Hz}, 1 \mathrm{H}), 3.25(\mathrm{dd}, J=13.7$, $8.1 \mathrm{~Hz}, 1 \mathrm{H}), 3.21(\mathrm{dd}, J=13.7,6.5 \mathrm{~Hz}, 1 \mathrm{H}) ;{ }^{13} \mathrm{C}\left\{{ }^{1} \mathrm{H}\right\} \operatorname{NMR}(151$ $\left.\mathrm{MHz}, \mathrm{CDCl}_{3}\right) \delta 137.1,134.6,130.9(\mathrm{q}, J=32.4 \mathrm{~Hz}), 129,3,129,2$, 128.6, 127.6, $126.2(\mathrm{q}, J=3.8 \mathrm{~Hz}), 124.4(\mathrm{q}, J=3.8 \mathrm{~Hz}), 123.9(\mathrm{q}, J$ $=272.2 \mathrm{~Hz}), 41.9,39.5 ;{ }^{19} \mathrm{~F}$ NMR $\left(282 \mathrm{MHz}, \mathrm{CDCl}_{3}\right) \delta-62.73$; IR: 3066, 3035, 2933, 2243, 1599, 1495, 1451, 1330, 1166, 1126, 1075, 911, 792, 754, $701 \mathrm{~cm}^{-1}$; HRMS (ESI+): $\mathrm{m} / z[\mathrm{M}+\mathrm{Na}]^{+}$calcd for $\mathrm{C}_{16} \mathrm{H}_{12} \mathrm{NF}_{3} \mathrm{Na}$, 298.0814; found 298.0814.

3-(naphthalen-1-yl)-2-phenylpropanenitrile $(\mathbf{4 g}){ }^{26 \mathrm{~b}}$ Colorless oil; 107 $\mathrm{mg}(83 \%) ;{ }^{1} \mathrm{H}$ NMR $\left(400 \mathrm{MHz}, \mathrm{CDCl}_{3}\right) \delta 7.97-7.89(\mathrm{~m}, 2 \mathrm{H}), 7.82-$ $7.80(\mathrm{~m}, 1 \mathrm{H}), 7.59-7.50(\mathrm{~m}, 2 \mathrm{H}), 7.43-7.29(\mathrm{~m}, 7 \mathrm{H}), 4.18(\mathrm{dd}, J=$ 8.7, $6.7 \mathrm{~Hz}, 1 \mathrm{H}), 3.70-3.57(\mathrm{~m}, 2 \mathrm{H}) ;{ }^{13} \mathrm{C}\left\{{ }^{1} \mathrm{H}\right\}$ NMR $(101 \mathrm{MHz}$, $\left.\mathrm{CDCl}_{3}\right) \delta 135.8,134.1,132.4,131.4,129.4,129.3,128.5,128.4$, $128.3,127.5,126.7,125.9,125.6,122.7,120.6,39.7,39.0$ ppm.

2-phenyl-3-(pyridin-4-yl)propanenitrile $(\mathbf{4 h}) .{ }^{14}$ Yellowish oil; $73 \mathrm{mg}$ $(70 \%) ;{ }^{1} \mathrm{H}$ NMR $\left(600 \mathrm{MHz}, \mathrm{CDCl}_{3}\right) \delta 8.62-8.60(\mathrm{~m}, 1 \mathrm{H}), 7.65-$ $7.62(\mathrm{~m}, 1 \mathrm{H}), 7.37-7.36(\mathrm{~m}, 4 \mathrm{H}), 7.34-7.30(\mathrm{~m}, 1 \mathrm{H}), 7.23-7.20$ $(\mathrm{m}, 1 \mathrm{H}), 7.15-7.14(\mathrm{~m}, 1 \mathrm{H}), 4.50(\mathrm{dd}, J=9.3,6.6 \mathrm{~Hz}, 1 \mathrm{H}), 3.36(\mathrm{dd}$, $J=13.9,9.3 \mathrm{~Hz}, 1 \mathrm{H}), 3.30(\mathrm{dd}, J=13.9,6.6 \mathrm{~Hz}, 1 \mathrm{H}) ;{ }^{13} \mathrm{C}\left\{{ }^{1} \mathrm{H}\right\} \mathrm{NMR}$ $\left(151 \mathrm{MHz}, \mathrm{CDCl}_{3}\right) \delta 156.1,149.5,136.9,135.4,129.1,128.2,127.4$, 124.0, 120.5, 44.1, 37.3.

2-phenyl-3-(pyridin-2-yl)propanenitrile $(4 \mathrm{i}) .{ }^{10}$ Yellowish oil; $49 \mathrm{mg}$ $(47 \%) ;{ }^{1} \mathrm{H}$ NMR $\left(600 \mathrm{MHz}, \mathrm{CDCl}_{3}\right) \delta 8.61-8.59(\mathrm{~m}, 1 \mathrm{H}), 7.62-$ $7.60(\mathrm{~m}, 1 \mathrm{H}), 7.36-7.35(\mathrm{~m}, 4 \mathrm{H}), 7.34-7.29(\mathrm{~m}, 1 \mathrm{H}), 7.21-7.18$ $(\mathrm{m}, 1 \mathrm{H}), 7.13-7.12(\mathrm{~m}, 1 \mathrm{H}), 4.48(\mathrm{dd}, J=9.3,6.5 \mathrm{~Hz}, 1 \mathrm{H}), 3.35(\mathrm{dd}$, $J=13.9,9.3 \mathrm{~Hz}, 1 \mathrm{H}), 3.28(\mathrm{dd}, J=13.9,6.5 \mathrm{~Hz}, 1 \mathrm{H}) ;{ }^{13} \mathrm{C}\left\{{ }^{1} \mathrm{H}\right\} \mathrm{NMR}$ 
$\left(151 \mathrm{MHz}, \mathrm{CDCl}_{3}\right) \delta \quad 156.3,149.8,136.9,135.5,129.2,128.3,127.5$ 124.0, 122.5, 120.7, 44.3, 37.4.

3-(furan-2-yl)-2-phenylpropanenitrile $(4 \mathrm{j}) .{ }^{26 \mathrm{c}}$ Colorless oil; $62 \mathrm{mg}$ (63\%); ${ }^{1} \mathrm{H}$ NMR $\left(600 \mathrm{MHz}, \mathrm{CDCl}_{3}\right) \delta 7.39-7.25(\mathrm{~m}, 6 \mathrm{H}), 6.30-$ $6.29(\mathrm{~m}, 1 \mathrm{H}), 6.12-6.11(\mathrm{~m}, 1 \mathrm{H}), 4.15(\mathrm{dd}, J=8.6,6.5 \mathrm{~Hz}, 1 \mathrm{H}), 3.27$ $(\mathrm{dd}, J=15.0,8.6 \mathrm{~Hz}, 1 \mathrm{H}), 3.19-3.13(\mathrm{~m}, 1 \mathrm{H}) ;{ }^{13} \mathrm{C}\left\{{ }^{1} \mathrm{H}\right\}$ NMR $(151$ $\left.\mathrm{MHz}, \mathrm{CDCl}_{3}\right) \delta 150.1,142.3,135.0,129.2,128.7,128.5,110.6$, $108.4,37.2,34.8$.

2-phenyl-3-(thiophen-2-yl)propanenitrile $(\mathbf{4 k}) .{ }^{18}$ Colorless oil; $87 \mathrm{mg}$ $(82 \%), ;{ }^{1} \mathrm{H}$ NMR $\left(600 \mathrm{MHz}, \mathrm{CDCl}_{3}\right) \delta 7.40-7.26(\mathrm{~m}, 5 \mathrm{H}), 7.17(\mathrm{~m}$, $1 \mathrm{H}), 6.92(\mathrm{~m}, 1 \mathrm{H}), 6.85(\mathrm{~m}, 1 \mathrm{H}), 4.04(\mathrm{dd}, J=8.1,6.4 \mathrm{~Hz}, 1 \mathrm{H}), 3.43$ (dd, $J=14.8,8.2 \mathrm{~Hz}, 1 \mathrm{H}), 3.34(\mathrm{dd}, J=14.8,6.3 \mathrm{~Hz}, 1 \mathrm{H}) ;{ }^{13} \mathrm{C}\left\{{ }^{1} \mathrm{H}\right\}$ NMR $\left(151 \mathrm{MHz}, \mathrm{CDCl}_{3}\right) \delta 138.0,134.8,129.2,128.5,127.5,127.2$, 127.1, 125.0, 40.1, 36.2.

2-phenylbutanenitrile (4l). ${ }^{12 \mathrm{a}}$ Colorless oil; $38 \mathrm{mg}(52 \%) ;{ }^{1} \mathrm{H}$ NMR $\left(600 \mathrm{MHz}, \mathrm{CDCl}_{3}\right) \delta 7.41-7.29(\mathrm{~m}, 5 \mathrm{H}), 3.74(\mathrm{t}, J=7.2 \mathrm{~Hz}, 1 \mathrm{H})$, $1.97-1.92(\mathrm{~m}, 2 \mathrm{H}), 1.08(\mathrm{t}, J=7.4 \mathrm{~Hz}, 3 \mathrm{H}) ;{ }^{13} \mathrm{C}\left\{{ }^{1} \mathrm{H}\right\}$ NMR $(151$ $\left.\mathrm{MHz}, \mathrm{CDCl}_{3}\right) \delta 135.9,129.1,128.1,127.4,120.9,39.0,29.4,11.6$

2-phenyloctanenitrile (4m). ${ }^{10}$ Colorless oil; $94 \mathrm{mg}(93 \%) ;{ }^{1} \mathrm{H}$ NMR $\left(600 \mathrm{MHz}, \mathrm{CDCl}_{3}\right) \delta 7.39-7.37(\mathrm{~m}, 2 \mathrm{H}), 7.34-7.31(\mathrm{~m}, 3 \mathrm{H}), 3.77$ $(\mathrm{dd}, J=8.7,6.2 \mathrm{~Hz}, 1 \mathrm{H}), 1.97-1.82(\mathrm{~m}, 2 \mathrm{H}), 1.55-1.42(\mathrm{~m}, 2 \mathrm{H})$, $1.36-1.25(\mathrm{~m}, 6 \mathrm{H}), 0.88(\mathrm{t}, J=6.9 \mathrm{~Hz}, 3 \mathrm{H}) ;{ }^{13} \mathrm{C}\left\{{ }^{1} \mathrm{H}\right\}$ NMR $(151$ $\left.\mathrm{MHz}, \mathrm{CDCl}_{3}\right) \delta$ 136.2, 129.1, 128.0, 127.3, 121.0, 37.5, 36.0, 31.6, 28.7, 27.0, 22.6, 14.1 .

4-methyl-2-phenylpentanenitrile $(\mathbf{4 n}){ }^{13 \mathrm{c}}$ Colorless oil; $71 \mathrm{mg}(82 \%)$; ${ }^{1} \mathrm{H}$ NMR $\left(600 \mathrm{MHz}, \mathrm{CDCl}_{3}\right) \delta 7.40-7.30(\mathrm{~m}, 5 \mathrm{H}), 3.81(\mathrm{dd}, J=9.8$, $6.4 \mathrm{~Hz}, 1 \mathrm{H}), 1.91$ (ddd, $J=13.4,9.8,5.5 \mathrm{~Hz}, 1 \mathrm{H}), 1.88-1.80(\mathrm{~m}$, $1 \mathrm{H}), 1.64$ (ddd, $J=13.3,8.4,6.3 \mathrm{~Hz}, 1 \mathrm{H}), 0.99(\mathrm{dd}, J=8.0,6.5 \mathrm{~Hz}$, $6 \mathrm{H}) ;{ }^{13} \mathrm{C}\left\{{ }^{1} \mathrm{H}\right\}$ NMR $\left(151 \mathrm{MHz}, \mathrm{CDCl}_{3}\right) \delta 136.4,129.2,128.1,127.3$, $121.1,45.1,35.61,26.2,22.7,21.7$

2-phenylhept-6-enenitrile (4o). ${ }^{26 \mathrm{~d}}$ Colorless oil; $78 \mathrm{mg}(78 \%) ;{ }^{1} \mathrm{H}$ $\operatorname{NMR}\left(600 \mathrm{MHz}, \mathrm{CDCl}_{3}\right) \delta \delta 7.41-7.30(\mathrm{~m}, 5 \mathrm{H}), 5.76(\mathrm{ddt}, J=17.0$, $10.2,6.7 \mathrm{~Hz}, 1 \mathrm{H}), 5.05-4.97(\mathrm{~m}, 2 \mathrm{H}), 3.79(\mathrm{dd}, J=8.6,6.2 \mathrm{~Hz}, 1 \mathrm{H})$, $2.13-2.08(\mathrm{~m}, 2 \mathrm{H}), 1.98-1.84(\mathrm{~m}, 2 \mathrm{H}), 1.67-1.52(\mathrm{~m}, 2 \mathrm{H})$ ${ }^{13} \mathrm{C}\left\{{ }^{1} \mathrm{H}\right\}$ NMR $\left(151 \mathrm{MHz}, \mathrm{CDCl}_{3}\right) \delta 137.6,136.0,129.1,128.1,127.3$, $120.9,115.5,37.3,35.3,33.0,26.2$.

3-cyclohexyl-2-phenylpropanenitrile $(4 p){ }^{14}$

Colorless oil; $77 \mathrm{mg}(72 \%) ;{ }^{1} \mathrm{H}$ NMR $\left(600 \mathrm{MHz}, \mathrm{CDCl}_{3}\right) \delta 7.40-$ $7.30(\mathrm{~m}, 5 \mathrm{H}), 3.85(\mathrm{dd}, J=10.1,6.2 \mathrm{~Hz}, 1 \mathrm{H}), 1.92-1.81(\mathrm{~m}, 2 \mathrm{H})$, $1.78-1.62(\mathrm{~m}, 5 \mathrm{H}), 1.57-1.49(\mathrm{~m}, 1 \mathrm{H}), 1.31-1.13(\mathrm{~m}, 3 \mathrm{H}), 1.01-$ $0.92(\mathrm{~m}, 2 \mathrm{H}) ;{ }^{13} \mathrm{C}\left\{{ }^{1} \mathrm{H}\right\}$ NMR $\left(151 \mathrm{MHz}, \mathrm{CDCl}_{3}\right) \delta$ 136.6, 129.2, $128.0,127.3,121.2,43.8,35.4,34.9,33.4,32.4,26.4,26.0,25.9$.

\section{General procedure of $\boldsymbol{C}$-methylation of nitriles}

A glass Ace pressure tube $(22 \mathrm{~mL})$ equipped with a magnetic stir bar was charged with Mn-2 (8 mg, $0.025 \mathrm{mmol}), \mathrm{Cs}_{2} \mathrm{CO}_{3}(16.3 \mathrm{mg}, 0.05$ $\mathrm{mmol}$ ). A rubber septum was attached to the tube and the reaction vessel was evacuated and backfilled with argon for three times. Under an inert atmosphere nitrile $(0.5 \mathrm{mmol})$, methanol $(1 \mathrm{~mL})$ and $1,4-$ dioxane $(1 \mathrm{~mL})$ were added and the tube was closed with a screw cap. The resulting mixture was stirred at $135^{\circ} \mathrm{C}$ (aluminum block) for $24 \mathrm{~h}$ under argon atmosphere. Upon cooling down to room temperature the residue was directly purified by flash column chromatography on silica gel eluting with pentane:diethyl ether mixtures to give the pure desired product.

2-phenylpropanenitrile (5a). ${ }^{26 \mathrm{e}}$ Yellowish oil; $46 \mathrm{mg}(70 \%) ;{ }^{1} \mathrm{H}$ NMR $\left(600 \mathrm{MHz}, \mathrm{CDCl}_{3}\right) \delta$ 7.41-7.31 (m, 5H), $3.90(\mathrm{q}, J=7.3 \mathrm{~Hz}, 1 \mathrm{H}), 1.65$ $(\mathrm{d}, J=7.3 \mathrm{~Hz}, 3 \mathrm{H}) ;{ }^{13} \mathrm{C}\left\{{ }^{1} \mathrm{H}\right\} \mathrm{NMR}\left(151 \mathrm{MHz}, \mathrm{CDCl}_{3}\right) \delta 137.2,129.3$, 128.2, 126.8, 121.7, 31.4, 21.6.

2-(2-methoxyphenyl)propanenitrile $(5 \boldsymbol{b}) .{ }^{26 \mathrm{f}}$

Yellowish oil $52 \mathrm{mg}(65 \%)$; eluent mixture: pentane: diethyl ether $(10: 1(\mathrm{v} / \mathrm{v}))$; ${ }^{1} \mathrm{H}$ NMR $\left(600 \mathrm{MHz}, \mathrm{CDCl}_{3}\right) \delta$ 7.43-7.41 (m, 1H), 7.32$7.29(\mathrm{~m}, 1 \mathrm{H}), 7.01-6.98(\mathrm{~m}, 1 \mathrm{H}), 6.91-6.89(\mathrm{~m}, 1 \mathrm{H}), 4.25(\mathrm{q}, J=7.2$ $\mathrm{Hz}, 1 \mathrm{H}), 3.87(\mathrm{~s}, 3 \mathrm{H}), 1.58(\mathrm{~d}, J=7.2 \mathrm{~Hz}, 3 \mathrm{H}) ;{ }^{13} \mathrm{C}\left\{{ }^{1} \mathrm{H}\right\}$ NMR $(151$ $\left.\mathrm{MHz}, \mathrm{CDCl}_{3}\right) \delta 156.2,129.4,127.7,125.5,122.1,121.1,110.9,55.6$, 25.7, 19.6 . 2-(3-methoxyphenyl)propanenitrile $(5 \mathrm{c}){ }^{26 \mathrm{~g}}$ Yellowish oil $56 \mathrm{mg}$ $(70 \%) ;{ }^{1} \mathrm{H}$ NMR $\left(600 \mathrm{MHz}, \mathrm{CDCl}_{3}\right) \delta 7.30(\mathrm{~m}, 1 \mathrm{H}), 6.94-6.93(\mathrm{~m}$, $1 \mathrm{H}), 6.89(\mathrm{~m}, 1 \mathrm{H}), 6.87-6.85(\mathrm{~m}, 1 \mathrm{H}), 3.87(\mathrm{q}, J=7.3 \mathrm{~Hz}, 1 \mathrm{H}), 3.83$ $(\mathrm{s}, 3 \mathrm{H}), 1.64(\mathrm{~d}, J=7.4 \mathrm{~Hz}, 3 \mathrm{H}) ;{ }^{13} \mathrm{C}\left\{{ }^{1} \mathrm{H}\right\}$ NMR $\left(151 \mathrm{MHz}, \mathrm{CDCl}_{3}\right) \delta$ 160.2, 138.6, 130.3, 121.7, 119.1, 113.5, 112.7, 55.5, 31.4, 21.5.

2-(4-methoxyphenyl)propanenitrile $(\mathbf{5 d}){ }^{26 \mathrm{e}}$ Yellowish oil $48 \mathrm{mg}$ $(59 \%) ;{ }^{1} \mathrm{H}$ NMR $\left(600 \mathrm{MHz}, \mathrm{CDCl}_{3}\right) \delta$ 7.30-7.28 (m, 2H), 6.94-6.91 $(\mathrm{m}, 2 \mathrm{H}), 3.87(\mathrm{q}, J=7.4 \mathrm{~Hz}, 1 \mathrm{H}), 3.83(\mathrm{~s}, 3 \mathrm{H}), 1.64(\mathrm{~d}, J=7.3 \mathrm{~Hz}$, $3 \mathrm{H}) ;{ }^{13} \mathrm{C}\left\{{ }^{1} \mathrm{H}\right\}$ NMR $\left(151 \mathrm{MHz}, \mathrm{CDCl}_{3}\right) \delta 159.4,129.2,128.0,122.0$, 114.6, 55.5, 30.6, 21.7.

2-(2-chlorophenyl)propanenitrile $(5 \boldsymbol{e}) .{ }^{26 \mathrm{e}}$ Yellowish oil; $51 \mathrm{mg}(61 \%)$; ${ }^{1} \mathrm{H}$ NMR $\left(600 \mathrm{MHz}, \mathrm{CDCl}_{3}\right) \delta 7.58(\mathrm{~m}, 1 \mathrm{H}), 7.41-7.39(\mathrm{~m}, 1 \mathrm{H}), 7.36-$ $7.32(\mathrm{~m}, 1 \mathrm{H}), 7.30-7.27(\mathrm{~m}, 1 \mathrm{H}), 4.36(\mathrm{q}, J=7.1 \mathrm{~Hz}, 1 \mathrm{H}), 1.63(\mathrm{~d}, J=$ $7.1 \mathrm{~Hz}, 3 \mathrm{H}) ;{ }^{13} \mathrm{C}\left\{{ }^{1} \mathrm{H}\right\} \mathrm{NMR}\left(151 \mathrm{MHz}, \mathrm{CDCl}_{3}\right) \delta 134.9,132.6,130.2$, $129.6128 .4,127.9,121.2,29.0,20.1$.

2-(4-chlorophenyl)propanenitrile (5f). ${ }^{26 \mathrm{e}}$ Yellowish oil; $57 \mathrm{mg}(69 \%)$; ${ }^{1} \mathrm{H}$ NMR $\left(600 \mathrm{MHz}, \mathrm{CDCl}_{3}\right) \delta$ 7.38-7.35 (m, 2H), 7.31-7.28 (m, 2H), $3.88(\mathrm{q}, J=7.4 \mathrm{~Hz}, 1 \mathrm{H}), 1.63(\mathrm{~d}, J=7.3 \mathrm{~Hz}, 3 \mathrm{H}) ;{ }^{13} \mathrm{C}\left\{{ }^{1} \mathrm{H}\right\} \mathrm{NMR}(151$ $\left.\mathrm{MHz}, \mathrm{CDCl}_{3}\right) \delta 135.6,134.2,129.5,128.2,121.3,30.9,21.5$.

2 -(pyridin-2-yl) propanenitrile $(5 \mathrm{~g}){ }^{26 \mathrm{~h}}$

$36 \mathrm{mg}(55 \%)$, yellowish oil; ${ }^{1} \mathrm{H} \mathrm{NMR}\left(600 \mathrm{MHz}, \mathrm{CDCl}_{3}\right) \delta 8.62-8.61$ $(\mathrm{m}, 1 \mathrm{H}), 7.77-7.74(\mathrm{~m}, 1 \mathrm{H}), 7.48-7.47(\mathrm{~m}, 1 \mathrm{H}), 7.29-7.27(\mathrm{~m}, 1 \mathrm{H})$, $4.08(\mathrm{q}, J=7.4 \mathrm{~Hz}, 1 \mathrm{H}), 1.73(\mathrm{~d}, J=7.4 \mathrm{~Hz}, 3 \mathrm{H}) ;{ }^{13} \mathrm{C}\left\{{ }^{1} \mathrm{H}\right\} \mathrm{NMR}(151$ $\left.\mathrm{MHz}, \mathrm{CDCl}_{3}\right) \delta 156.2,150.0,137.6,123.1,121.2,121.1,33.9,19.8$. 2-(thiophen-2-yl)propanenitrile $(\mathbf{5 h}){ }^{26 \mathrm{i}}$ Yellowish oil; $42 \mathrm{mg}(61 \%)$; ${ }^{1} \mathrm{H}$ NMR $\left(600 \mathrm{MHz}, \mathrm{CDCl}_{3}\right) \delta$ 7.28-7.27 $(\mathrm{m}, 1 \mathrm{H}), 7.08-7.07(\mathrm{~m}, 1 \mathrm{H})$, $6.99-6.98(\mathrm{~m}, 1 \mathrm{H}), 4.18(\mathrm{q}, J=7.4 \mathrm{~Hz}, 1 \mathrm{H}), 1.74(\mathrm{~d}, J=7.2 \mathrm{~Hz}, 3 \mathrm{H})$; ${ }^{13} \mathrm{C}\left\{{ }^{1} \mathrm{H}\right\} \mathrm{NMR}\left(151 \mathrm{MHz}, \mathrm{CDCl}_{3}\right) \delta 139.3,127.2,125.7,125.5,120.8$, 26.7, 21.6.

Representative procedure for gram-scale synthesis of 2-phenyl-3(p-tolyl)propanenitrile (4c)

A glass pressure tube $(25 \mathrm{~mL})$ equipped with a magnetic stirrer was charged with Mn-2 (35.2 mg, $0.055 \mathrm{mmol}), \mathrm{Cs}_{2} \mathrm{CO}_{3}(179.3 \mathrm{mg}, 0.55$ $\mathrm{mmol}$ ). A rubber septum was attached to the tube and the reaction vessel was evacuated and backfilled with argon for three times. Under an inert atmosphere $p$-tolylmethanol $(1.34 \mathrm{~g}, 11.0 \mathrm{mmol}), \quad 2$ phenylacetonitrile $(632 \mu \mathrm{L}, 644 \mathrm{mg}, 5.5 \mathrm{mmol})$ and toluene $(5.5 \mathrm{~mL})$ were added and the tube was closed with a screw cap. The resulting mixture was stirred at $135^{\circ} \mathrm{C}$ (aluminum block) for $24 \mathrm{~h}$ under argon atmosphere. Upon cooling down to room temperature the residue was concentrated under reduced pressure and purified by flash column chromatography on silica gel eluting with pentane: diethyl ether (20:1 $(\mathrm{v} / \mathrm{v}))$ to give the pure $4 \mathrm{c}(1022 \mathrm{mg}, 84 \%$ yield $)$.

\section{ASSOCIATED CONTENT}

\section{Supporting Information}

The Supporting Information is available free of charge on the ACS Publications website: copies of NMR spectra (PDF)

\section{AUTHORS INFORMATION}

\section{Corresponding Authors}

* Magnus.Rueping@rwth-aachen.de

* Osama.Elsepelgy@rwth-aachen.de

\section{ORCID}

Magnus Rueping: 0000-0003-4580-5227

Osama El-Sepelgy: 0000-0003-3131-4988

Notes

The authors declare no competing financial interests.

\section{ACKNOWLEDGMENTS}

J.C.B. acknowledges the German Federal Environmental Foundation (DBU) for a doctoral fellowship. J.S. is grateful for a doctoral fellowship provided by the Fonds der Chemischen Industrie. 


\section{REFERENCES}

(1) Vispute, T. P., Zhang, H., Sanna, A., Xiao, R. Huber, G. W. Renewable Chemical Commodity Feedstocks from Integrated Catalytic Processing of Pyrolysis Oils. Science 2010, 330, 1222-1227.

(2) Selected Recent Reviews: (a) Corma, A.; Navas, J.; Sabater, M. J. Advances in One-Pot Synthesis through Borrowing Hydrogen Catalysis. Chem. Rev. 2018, 118, 1410. (b) Crabtree, R. H. Homogeneous Transition Metal Catalysis of Acceptorless Dehydrogenative Alcohol Oxidation: Applications in Hydrogen Storage and to Heterocycle Synthesis. Chem. Rev. 2017, 117, 92289246. (c) Chelucci, G. Ruthenium and osmium complexes in C-C bond-forming reactions by borrowing hydrogen catalysis. Coord. Chem. Rev. 2017, 331, 1-36. (d) Huang, F.; Liu, Z.; Yu, Z. CAlkylation of Ketones and Related Compounds by Alcohols: Transition-Metal-Catalyzed Dehydrogenation. Angew. Chem. Int. Ed. 2016, 55, 862-875. (e) Nandakumar, A., Midya, S. P., Landge, V. G. Balaraman, E. Transition-Metal-Catalyzed Hydrogen-Transfer Annulations: Access to Heterocyclic Scaffolds. Angew. Chem. Int. Ed. 2015, 54, 11022-11034. (f) Yang, Q.; Wang, Q.; Yu, Z. Substitution of alcohols by N-nucleophiles via transition metal-catalyzed dehydrogenation. Chem. Soc. Rev. 2015, 44, 2305-2329. (g) Obora, Y. Recent Advances in $\alpha$-Alkylation Reactions using Alcohols with Hydrogen Borrowing Methodologies. ACS Catal. 2014, 4, 3972-3981. (h) Gunanathan, C.; Milstein, D. Applications of Acceptorless Dehydrogenation and Related Transformations in Chemical Synthesis. Science 2013, 341, 249. (i) Guillena, G.; Ramln, D. J.; Yus, M. Hydrogen Autotransfer in the $\mathrm{N}$-Alkylation of Amines and Related Compounds using Alcohols and Amines as Electrophiles. Chem. Rev. 2010, 110, 1611.

(3) Bullock, R. M., Catalysis without Precious Metals. WileyVCH: Weinheim, 2010.

(4) (a) Bruneau-Voisine, A.; Pallova, L.; Bastin, S.; César, V.; Sortais, J.-B., Manganese catalyzed $\alpha$-methylation of ketones with methanol as a $\mathrm{C} 1$ source. Chem.Commun. 2019, 55, 314-317. (b) Sklyaruk, J.; Borghs, J.; El-Sepelgy, O.; Rueping, M., Catalytic $\mathrm{C}_{1}$ Alkylation with Methanol and Isotope Labelled Methanol. Angew. Chem. Int. Ed. 2018, 14, 775-779. (c) Barman, M. K.; Jana, A.; Maji, B., Phosphine-Free NNN-Manganese Complex Catalyzed $\alpha$ Alkylation of Ketones with Primary Alcohols and Friedlaender Quinoline Synthesis, Adv. Synth. Catal. 2018, 360, 3233-3238. (d) Zhang, G.; Wu, J.; Zeng, H.; Zhang, S.; Yin, Z.; Zheng, S. CobaltCatalyzed $\alpha$-Alkylation of Ketones with Primary Alcohols. Org. Lett. 2017, 19, 1080-1083. (e) Pena-Lopez, M.; Piehl, P.; Elangovan, S.; Neumann, H.; Beller, M. Manganese Catalyzed HydrogenAutotransfer C-C Bond Formation: $\alpha$-Alkylation of Ketones with Primary Alcohols. Angew. Chem. Int. Ed. 2016, 55, 14967-14971. (f) Elangovan, S.; Sortais, J.-B.; Beller, M.; Darcel, C. Iron-Catalyzed $\alpha$-Alkylation of Ketones with Alcohols. Angew. Chem. Int. Ed. 2015, 54, 14483-14486.

(5) (a) El-Sepelgy, O.; Matador, E.; Brzozowska, A.; Rueping, M., C-Alkylation of Secondary Alcohols by Primary Alcohols through Manganese-Catalyzed Double Hydrogen Autotransfer. ChemSusChem 2019, doi,10.1002/cssc.201801660. (b) Liu, T.; Wang, L.; Wu, K.; Yu, Z. Manganese-Catalyzed $\beta$-Alkylation of Secondary Alcohols with Primary Alcohols under Phosphine-Free Conditions. ACS Catal. 2018, 8, 7201-7207. (c) Kulkarni; N. V.; Brennessel; W. W.; Jones; W. D. Catalytic Upgrading of Ethanol to n-Butanol via Manganese-Mediated Guerbet Reaction. ACS Catal. 2018, 8 , 997-1002. (d) Fu, S.; Shao, Z.; Wang, Y.; Liu, Q. ManganeseCatalyzed Upgrading of Ethanol into 1-Butanol. J. Am. Chem. Soc.; 2017, 139, 11941-11948. (e) Freitag, F.; Irrgang, T.; Kempe, R. Cobalt-Catalyzed Alkylation of Secondary Alcohols with Primary Alcohols via Borrowing Hydrogen/Hydrogen Autotransfer. Chem. Eur. J. 2017, 23, 12110-12113.

(6) (a) Jang, Y. K.; Krückel, T.; Rueping, M.; El-Sepelgy, O., Sustainable Alkylation of Unactivated Esters and Amides with Alcohols Enabled by Manganese Catalysis. Org Lett. 2018, 20, 7779 7783. (b) Chakraborty, S.; Daw, P.; Ben David, Y.; Milstein, D., Manganese-Catalyzed $\alpha$-Alkylation of Ketones, Esters, and Amides Using Alcohols. ACS Catal. 2018, 8, 10300-10305. (c) Deibl, N.;
Kempe, R. General and Mild Cobalt-Catalyzed C-Alkylation of Unactivated Amides and Esters with Alcohols. J. Am. Chem. Soc. 2016, 138, 10786-10789.

(7) Werkmeister, S.; Bornschein, C.; Junge, K.; Beller, M. Selective Ruthenium-Catalyzed Transfer Hydrogenations of Nitriles to Amines with 2-Butanol. Chem. Eur. J. 2013, 19, 4437-4440.

(8) (a) Paul, B.; Shee, S.; Panja, D.; Chakrabarti, K.; Kundu, S. Direct Synthesis of N,N-Dimethylated and $\beta$-Methyl N,NDimethylated Amines from Nitriles Using Methanol: Experimental and Computational Studies. ACS Catal. 2018, 8, 2890-2896. (b) Werkmeister, S.; Bornschein, C.; Junge, K.; Beller, M. Ruthenium-Catalyzed Transfer Hydrogenation of Nitriles: Reduction and Subsequent N-Monoalkylation to Secondary Amines. Eur. J. Org. Chem. 2013, 3671-3674.

(9) (a) Kang, B.; Hong, S. H. Hydrogen Acceptor- and Base-Free N-Formylation of Nitriles and Amines using Methanol as C1 Source. Adv. Synth. Catal. 2015, 357, 834-840. (b) Kang, B., Fu, Z., Hong, S.H. Ruthenium-Catalyzed Redox-Neutral and Single-Step Amide Synthesis from Alcohol and Nitrile with Complete Atom Economy. J. Am. Chem. Soc., 2013, 135, 11704-11707.

(10) Li, F., Zou, X., Wang, N. Direct Coupling of Arylacetonitriles and Primary Alcohols to $\alpha$-Alkylated Arylacetamides with Complete Atom Economy Catalyzed by a Rhodium Complex-Triphenylphosphine- Potassium Hydroxide System. Adv. Synth. Catal. 2015, 357, 1405-1415.

(11) Chakraborty, S.; Das, U. K.; Ben-David, Y.; Milstein, D. Manganese Catalyzed $\alpha$-Olefination of Nitriles by Primary Alcohols. J. Am. Chem. Soc. 2017, 139, 11710-11713.

(12) (a) Thiyagarajan, S.; Gunanathan, C. Facile Ruthenium(II)-Catalyzed $\alpha$-Alkylation of Arylmethyl Nitriles Using Alcohols Enabled by Metal-Ligand Cooperation. ACS Catal. 2017, 7, 54835490. (b) Alós, J.; Bolaño, T.; Esteruelas, M. A.; Oliván, M.; Oñate, E.; Valencia, M., POP-Pincer Ruthenium Complexes: d6 Counterparts of Osmium d4 Species. Inorg. Chem. 2014, 53 , 11951209. (c) Kuwahara, T.; Fukuyama, T.; Ryu, I. Synthesis of Alkylated Nitriles by $[\mathrm{RuHCl}(\mathrm{CO})(\mathrm{PPh} 3) 3]-C a t a l y z e d$ Alkylation of Acetonitrile Using Primary Alcohols. Chem, Lett. 2013, 42, 1163-1165. (d) Cheung, H. W.; Li, J.; Zheng, W.; Zhou, Z.; Chiu, Y. H.; Lin, Z.; Lau, C. P. Dialkylamino Cyclopentadienyl Ruthenium(ii) ComplexCatalyzed $\alpha$-Alkylation of Arylacetonitriles with Primary Alcohols. Dalton Trans. 2010, 39, 265-274. (e) Motokura, K.; Fujita, N.; Mori, K.; Mizugaki, T.; Ebitani, K.; Jitsukawa, K.; Kaneda, K. Environmentally Friendly One-Pot Synthesis of $\alpha$-Alkylated Nitriles Using Hydrotalcite-Supported Metal Species as Multifunctional Solid Catalysts. Chem. Eur. J. 2006, 12, 8228-8239. (f) Motokura, K.; Nishimura, D.; Mori, K.; Mizugaki, T.; Ebitani, K.; Kaneda, K. A. Ruthenium-Grafted Hydrotalcite as a Multifunctional Catalyst for Direct $\alpha$ Alkylation of Nitriles with Primary Alcohols. J. Am. Chem. Soc. 2004, 126, 5662-5663. (g) Zhu, Z-H.; Li, Y.; Wang, Y-B.; Lan, Xinju, Z-G.; Zhu, X.; Hao, X-Q; Song; M.-P. $\alpha$-Alkylation of Nitriles with Alcohols Catalyzed by NNN'Pincer Ru(II) Complexes Bearing Bipyridyl Imidazoline Ligands. Organometallics 2019, 38, 2156.

(13) (a) Sawaguchi, T.; Obora, Y. Iridium Catalyzed $\alpha$ Alkylation of Acetonitrile with Primary and Secondary Alcohols. Chem, Lett. 2011, 40, 1055-1057. (b) Anxionnat, B.; Gomez Pardo, D.; Ricci, G.; Cossy, J. Monoalkylation of Acetonitrile by Primary Alcohols Catalyzed by Iridium Complexes. Org. Lett. 2011, 13, 40844087. (c) Morita, M.; Obora, Y.; Ishii, Y., Alkylation of active methylene compounds with alcohols catalyzed by an iridium complex. Chem. Commun.2007, 2850-2852. (d) Löfberg, C.; Grigg, R.; Whittaker, M. A.; Keep, A.; Derrick, A. Efficient Solvent-Free Selective Monoalkylation of Arylacetonitriles with Mono-, Bis-, and Tris-primary Alcohols Catalyzed by a Cp*Ir Complex. J. Org. Chem. 2006, 71, 8023-8027. (e) Ishii, Y.; Iwahama, Takahiro; N., Tatsuya Preparation of nitriles by $\alpha$-alkylation by alcohols, 2005 JP 2005-64657.

(14) (a) Junjun, L.; Yuxuan, L.; Weijun, T.; Dong, X.; Chaoqun, L.; Jianliang, X.; Chao, W. Atmosphere-Controlled Chemoselectivity: Rhodium-Catalyzed Alkylation and Olefination of Alkylnitriles with Alcohols. Chem. Eur. J. 2017, 23, 14445- 14449. 
(15) Buil, M. L.; Esteruelas, M. A.; Herrero, J.; Izquierdo, S.; Pastor, I. M.; Yus, M. Osmium Catalyst for the Borrowing Hydrogen Methodology: $\alpha$-Alkylation of Arylacetonitriles and Methyl Ketones. ACS Catal. 2013, 3, 2072-2075.

(16) Corma, A.; Rjdenas, T.; Sabater, M. J. Monoalkylations with alcohols by a cascade reaction on bifunctional solid catalysts: Reaction kinetics and mechanism. J. Catal. 2011, 279, 319- 327.

(17) (a) López R.; Palomo C. Cyanoalkylation: Alkylnitriles in Catalytic C-C Bond Forming Reactions. Angew. Chem. Int. Ed. 2015, 54, $13170-13184$ and the references cited in (b) Rappoport, Z.; Patai S.; Chemistry of the Cyano Group, Wiley, London, 1970.

(18) Ma, W.; Cui, S.; Sun, H.; Tang, W.; Xue, D.; Li, C.; Fan, J.; Xiao, J.; Wang, C. Iron Catalyzed Alkylation of Nitriles with Alcohols. Chem. - A Eur. J. 2018, 24, 13118-13123.

(19) (a) El-Sepelgy, O.; Alandini, N.; Rueping M. Merging Iron Catalysis and Biocatalysis - Iron Carbonyl Complexes as Efficient Hydrogen Autotransfer Catalysts in Dynamic Kinetic Resolutions. Angew. Chem. Int. Ed. 2016, 55, 13602-13605. (b) El-Sepelgy, O.; Brzozowska, A.; Azofra, L. M.; Jang, Y. K.; Cavallo, L.; Rueping, M. Experimental and Computational Study of an Unexpected IronCatalyzed Carboetherification by Cooperative Metal and Ligand Substrate Interaction and Proton Shuttling. Angew. Chem. Int. Ed. 2017, 56, 14863-14867. (c) El-Sepelgy, O.; Brzozowska, A.; Rueping, M. Asymmetric Chemoenzymatic Reductive Acylation of Ketones by a Combined Iron-Catalyzed Hydrogenation-Racemization and Enzymatic Resolution Cascade. ChemSusChem 2017, 10, 1664 1668. (d) El-Sepelgy, O.; Brzozowska, A.; Sklyaruk, J.; Jang, Y. K.; Zubar, V.; Rueping, M. Cooperative Metal-Ligand Catalyzed Intramolecular Hydroamination and Hydroalkoxylation of Allenes Using a Stable Iron Catalyst. Org. Lett. 2018, 20, 696-679.

(20) Mn-catalyzed hydrogenation of polar bonds, seminal example: (a) Elangovan, S.; Topf, C.; Fischer, S.; Jiao, H.; Spannenberg, A.; Baumann, W.; Ludwig, R.; Junge, K.; Beller, M., Selective Catalytic Hydrogenations of Nitriles, Ketones, and Aldehydes by WellDefined Manganese Pincer Complexes. J. Am. Chem. Soc. 2016, 138, 8809 - 8814. Example from our group: (b) Zubar, V.; Lebedev, Y.; Azofra, L. M.; Cavallo, L.; El-Sepelgy, O.; Rueping, M., Hydrogenation of CO2-Derived Carbonates and Polycarbonates to Methanol and Diols by Metal-Ligand Cooperative Manganese Catalysis. Angew. Chem. Int. Ed. 2018, 57, $13439-13443$.

(21) Mn-catalyzed hydrogenation of non-polar bonds: (a) Brzozowska, A.; Azofra, L. M.; Zubar, V.; Atodiresei, I; Cavallo, L.; Rueping, M.; El-Sepelgy, O. Highly Chemo- and Stereoselective Transfer Semihydrogenation of Alkynes Catalyzed by a Stable, WellDefined Manganese(II) Complex. ACS Catal. 2018, 8, 4103-4109. (b) Zhou, Y. P.; Mo, Z.; Luecke, M. P.; Driess, M., Stereoselective Transfer Semi-Hydrogenation of Alkynes to E-Olefins with $\mathrm{N}$ Heterocyclic Silylene-Manganese Catalysts. Chem. Eur. J. 2018, 24, 4780- 4784.

(22) For recent reviews on Mn-catalysis: Recent Reviews: (a) Zell, T.; Langer, R., From Ruthenium to Iron and Manganese-A Mechanistic View on Challenges and Design Principles of BaseMetal Hydrogenation Catalysts. ChemCatChem 2018, 10, 1930-1940; (b) Mukherjee, A.; Milstein, D., Homogeneous Catalysis by Cobalt and Manganese Pincer Complexes. ACS Catal 2018, 8, 11435-11469. (c) Kallmeier, F.; Kempe, R., Manganese Complexes for (De)Hydrogenation Catalysis: A Comparison to Cobalt and Iron Catalysts. Angw. Chem. Int. Ed. 2018, 57 , 46-60. (d) Gorgas, N.; Kirchner, K., Isoelectronic Manganese and Iron Hydrogenation/Dehydrogenation Catalysts: Similarities and Divergences. Acc. Chem.Res. 2018, 51, 1558-1569. (e) Filonenko, G. A.; van Putten, R.; Hensen, E. J. M.; Pidko, E. A., Catalytic (de)hydrogenation promoted by non-precious metals - $\mathrm{Co}, \mathrm{Fe}$ and $\mathrm{Mn}$ : recent advances in an emerging field. Chem. Soc. rev. 2018, 47, 1459-1483. (f) Maji, B.; Barman, M. K., Recent Developments of Manganese Complexes for Catalytic Hydrogenation and Dehydrogenation Reactions. Synthesis 2017, 49, 3377-3393. (g) Garbe, M.; Junge, K.; Beller, M., Homogeneous Catalysis by Manganese-Based Pincer Complexes. Eur. J. Org. Chem. 2017, 4344-4362. (h) Valyaev, D. A.; Lavigne, G.; Lugan, N., Manganese organometallic compounds in homogeneous catalysis: Past, present, and prospects. Coord. Chem. Rev. 2016, 308, 191-235. (i) Carney, J. R.; Dillon, B. R.; Thomas, S. P., Recent Advances of Manganese Catalysis for Organic Synthesis. Eur. J. Org. Chem. 2016, 3912-3929. For recent examples from our group: (j) Borghs, J. C.; Azofra, L. M.; Biberger, T.; Linnenberg, O.; Cavallo, L.; Rueping, M.; El-Sepelgy, O., Manganese-Catalyzed Multicomponent Synthesis of Pyrroles through Acceptorless Dehydrogenation Hydrogen Autotransfer Catalysis: Experiment and Computation. ChemSusChem 2019, DOI:10.1002/cssc.201802416. (k) Borghs, J. C.; Lebedev, Y.; Rueping, M.; El-Sepelgy, O., Sustainable Manganese-Catalyzed Solvent-Free Synthesis of Pyrroles from 1,4-Diols and Primary Amines. Org. Lett. 2019, 21, 70-74.

(23) Jana, A.; Reddy, C. B.; Maji B. Manganese Catalyzed $\alpha$ Alkylation of Nitriles with Primary Alcohols ACS Catal., 2018, 8, 9226-9231.

(24) Liu, W.; Ackermann, L. Manganese-Catalyzed C-H Activation. ACS Catal. 2016, 6, 3743-3752.

(25) For a recent detailed DFT study: (a) Luque-Urrutia, J. A.; Solà, M.; Milstein, D.; Poater, A., Mechanism of the ManganesePincer-Catalyzed Acceptorless Dehydrogenative Coupling of Nitriles and Alcohols. J. Am. Chem. Soc. 2019, 141, 2398-2403. (b) Lu, Y.; Zhao, R.; Guo, J.; Liu, Z.; Dagnaw, W. M.; Wang, Z., A Unified Mechanism to Account for Manganese or Ruthenium Catalyzed Nitrile $\alpha$-Olefinations by Primary or Secondary Alcohols: A DFT Mechanistic Study. Chem. - A Eur. J. 2019, 25, 3939-3949. For related observations: (c) Vogt, M.; Nerush, A.; Iron, M. A.; Leitus, G.; Diskin-Posner, Y.; Shimon, L. J. W.; Ben-David, Y.; Milstein, D. Activation of Nitriles by Metal Ligand Cooperation. Reversible Formation of Ketimido- and Enamido-Rhenium PNP Pincer Complexes and Relevance to Catalytic Design J. Am. Chem. Soc. 2013, 135, 17004-17018. (d) Perdriau, S.; Zijlstra, D. S.; Heeres, H. J.; de Vries, J. G.; Otten, E. A Metal-Ligand Cooperative Pathway for Intermolecular Oxa-Michael Additions to Unsaturated Nitriles. Angew. Chem., Int. Ed. 2015, 54, 4236 - 4240. (e) Nerush, A.; Vogt, M.; Gellrich, U.; Leitus, G.; Ben-David, Y.; Milstein D. Template Catalysis by MetalLigand Cooperation. $\mathrm{C}-\mathrm{C}$ Bond Formation via Conjugate Addition of Non-activated Nitriles under Mild, Base-free Conditions Catalyzed by a Manganese Pincer Complex. J. Am. Chem. Soc., 2016, 138, 69856997.

(26) (a) Shang, R.; Ji, D.-S.; Chu, L.; Fu, Y.; Liu, L. Synthesis of $\alpha$-Aryl Nitriles through PalladiumCatalyzed Decarboxylative Coupling of Cyanoacetate Salts with Aryl Halides and Triflates. Angew. Chem. Int. Ed. 2011, 50, 4470-4474. (b) Xiao, J.; Yang, J.; Chen, T.; Hana, L.-B. Nickel Catalyzed $\alpha$-Benzylation of Arylacetonitriles via C-O Activation. Adv.Synth. Catal. 2016, 358, 816-819. (c) Kulp, S. S.; Caldwell, C. B. Reduction of alpha, betadiarylacrylonitriles by sodium borohydride. J. Org. Chem. 1980, 45, 171-173 (d) Choi, J.; Fu, G. C. Catalytic Asymmetric Synthesis of Secondary Nitriles via Stereoconvergent Negishi Arylations and Alkenylations of Racemic $\alpha$-Bromonitriles. J. Am. Chem. Soc. 2012, 134, 9102-9105. (e) Huang, Y.; Yu, Y.; Zhu, Z.; Zhu, C.; Cen, J.; Li, $\mathrm{X}$; Wu, W.; Jiang, H. Copper-Catalyzed Cyanation of $\mathrm{N}$ Tosylhydrazones with Thiocyanate Salt as the "CN" Source. J. Org. Chem. 2017, 82, 7621-7627. (f) Chen, G.; Wang, Z.; Wu, J.; Ding, K. Facile Preparation of $\alpha$-Aryl Nitriles by Direct Cyanation of Alcohols with TMSCN Under the Catalysis of InX3. Org. Lett. 2008, 10, 4573 4576. (g) Cheng, A.; Uyeno, E.; Polgar, W.; Toll, L.; Lawson, J. A.; DeGraw, J. I.; Loew, G.; Camerman, A.; Camerman, N. NSubstituent modulation of opiate agonist/antagonist activity in resolved 3-methyl-3-(m-hydroxyphenyl)piperidines. J. Med. Chem. 1986, 29, 531-537. (h) Möhrle, H.; Pycior, M. 2-(Pyridyl)-propionitriles and their Aminomethylation. Arch. Pharm. (Weinheim) 1995, 328, 293-296. (i) Guin, J.; Varseev, G.; List, B. Catalytic Asymmetric Protonation of Silyl Ketene Imines. J. Am. Chem. Soc. 2013, 135, 2100-2103. 


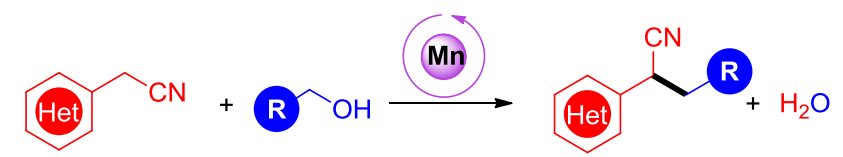

readily abandant feedstocks

value-added alkyl nitriles

*First row metal catalyst * Catalytic amount of base * Methanol as C1 source * High yield and excellent selectivity 